\title{
GRASS: GRAph Spectral Sparsification Leveraging Scalable Spectral Perturbation Analysis
}

\author{
Zhuo Feng, Senior Member, IEEE
}

\begin{abstract}
Spectral graph sparsification aims to find ultrasparse subgraphs whose Laplacian matrix can well approximate the original Laplacian eigenvalues and eigenvectors. In recent years, spectral sparsification techniques have been extensively studied for accelerating various numerical and graph-related applications. Prior nearly-linear-time spectral sparsification methods first extract low-stretch spanning tree from the original graph to form the backbone of the sparsifier, and then recover small portions of spectrally-critical off-tree edges to the spanning tree to significantly improve the approximation quality. However, it is not clear how many off-tree edges should be recovered for achieving a desired spectral similarity level within the sparsifier. Motivated by recent graph signal processing techniques, this paper proposes a similarity-aware spectral graph sparsification framework that leverages efficient spectral off-tree edge embedding and filtering schemes to construct spectral sparsifiers with guaranteed spectral similarity (relative condition number) level. An iterative graph densification scheme is also introduced to facilitate efficient and effective filtering of off-tree edges for highly ill-conditioned problems. The proposed method has been validated using various kinds of graphs obtained from public domain sparse matrix collections relevant to VLSI CAD, finite element analysis, as well as social and data networks frequently studied in many machine learning and data mining applications. For instance, a sparse SDD matrix with 40 million unknowns and 180 million nonzeros can be solved (1E-3 accuracy level) within two minutes using a single CPU core and about $6 G B$ memory.
\end{abstract}

Index Terms-Spectral graph theory, iterative matrix solver, graph partitioning, circuit analysis, perturbation analysis

\section{INTRODUCTION}

Spectral methods are playing increasingly important roles in many graph and numerical applications [32], such as scientific computing [30], numerical optimization [5], data mining [24], graph analytics [16], [18], [42], machine learning [9], [10], [34], graph signal processing [26], and VLSI computer-aided design [12], [39], [40]. For example, classical spectral graph partitioning (data clustering) algorithms embed original graphs into low-dimensional space using the first few nontrivial eigenvectors of graph Laplacians and subsequently perform graph partitioning (data clustering) on the low-dimensional graphs to obtain high-quality solution [24], [33]. To further push the limit of spectral methods for large graphs, mathematics and theoretical computer science researchers have extensively studied many theoretically-sound research problems related to spectral graph theory. Recent spectral graph sparsification research [2], [6], [20], [24], [27], [29] allows computing nearly-linear-sized subgraphs (sparsifiers) that can robustly

The author is with the Department of ECE, Stevens Institute of Technology, Hoboken, NJ, 07030. Email: zfeng12@stevens.edu. This work is supported in part by the National Science Foundation under Grants CCF-1350206 (CAREER), CCF-1318694 (SHF), CCF-1909105 (SHF), CCF-1618364 (SHF), and a gift from Keysight Technologies. preserve the spectra (i.e., eigenvalues and eigenvectors) of the original graph's Laplacian, which immediately leads to a series of theoretically nearly-linear-time numerical and graph algorithms for solving sparse matrices, graph-based semisupervised learning (SSL), spectral graph partitioning (data clustering), and max-flow problems [5], [19], [29], [30]. For example, sparsified circuit networks allow for developing more scalable computer-aided (CAD) design algorithms for designing large VLSI systems [12], [39]; sparsified social (data) networks enable to more efficiently understand and analyze large social (data) networks [32]; sparsified matrices can be immediately leveraged to accelerate the solution computation of large linear system of equations [41]. To this end, a spectral sparsification algorithm leveraging an edge sampling scheme that sets sampling probabilities proportional to edge effective resistances (of the original graph) has been proposed in [27]. However, it becomes a chicken-and-egg problem since calculating effective resistances (leverage scores for edge sampling) requires solving the original graph Laplacian matrix multiple times (even when using JohnsonLindenstrauss (JL) lemma [27]) and thus can be extremely expensive for very large graphs.

This paper aims to address the standing question whether there exists a practically-efficient, nearly-linear time spectral graph sparsification algorithm that can immediately enable the development of nearly-linear time sparse SDD matrix solvers and other graph-based algorithms for large-scale, real-world problems. Our work is built upon the recent spectral perturbation analysis framework that allows for highly-scalable spectral sparsification of large (weighted) undirected graphs [12], [13], which also shows connections with the CourantFishcher theorem for generalized eigenvalues as well as recent graph signal processing techniques [26].

Our method starts by extracting a spectrally-critical spanning tree subgraph as a backbone of the sparsifier, and subsequently recovers a small portion spectrally-critical offtree edges to the spanning tree. In many scientific computing and graph-related applications, it is usually desired to construct sparsifiers according to a given spectral similarity level: introducing too few edges may lead to poor approximation of the original graph, whereas too many edges can result in rather high computational complexity. For example, when using a preconditioned conjugate gradient (PCG) method to solve a symmetric diagonally dominant (SDD) matrix for multiple right-hand-side (RHS) vectors, it is hoped the PCG solver would converge to a good solution as fast as possible, which usually requires the sparsifier (preconditioner) to be highly spectrally-similar to the original problem or achieve relatively a small condition number; on the other hand, in many graph 
partitioning tasks, only the Fiedler vector (the first nontrivial eigenvector) of graph Laplacian is needed [28], so even a sparsifier with a much lower spectral similarity should suffice. To this end, this work introduces a similarity-aware spectral graph sparsification framework that leverages efficient spectral off-tree edge embedding and filtering schemes to construct spectral sparsifiers with guaranteed spectral similarity. The contribution of this work has been summarized as follows:

1) We present a nearly-linear time yet practically-efficient framework for constructing ultra-sparsifier subgraph from a spanning tree subgraph via efficient spectral perturbation analysis of generalized eigenvalue problem. The proposed algorithm allows effectively fixing the largest generalized eigenvalues, by recovering the most spectrally-critical off-tree edges to the spanning-tree subgraph.

2) We present a similarity-aware spectral sparsification framework by leveraging scalable spectral off-tree edge embedding and filtering schemes motivated by recent graph signal processing techniques [26]. Such a scheme enables to flexibly trade off the complexity and spectral similarity of the sparsified graph.

3) For highly ill-conditioned problems, we introduce an iterative graph densification scheme to more effectively fix the most problematic eigenvalues by progressively improving the ultra-sparsifier subgraphs. Compared to the single-pass spectral sparsification scheme, the proposed scheme can always achieve greater reduction of relative condition numbers, thereby leading to more effective spectral approximation of the original graph Laplacian.

4) Extensive experiments have been conducted to validate the proposed method in various numerical and graphrelated applications, such as solving sparse SDD matrices, spectral graph partitioning, as well as compression of large social and data networks. Very promising results for even extremely ill-conditioned VLSI, thermal and finite element analysis problems have been obtained.

The rest of this paper is organized as follows. Section II provides a brief introduction to graph Laplacians and the state of the art in spectral sparsification of graphs. In Sections III and IV] a scalable similarity-aware spectral sparsification method based on spectral perturbation analysis is described in detail. Section $\mathrm{V}$ demonstrates extensive experimental results for a variety of real-world, large-scale sparse Laplacian matrix and graph problems, which is followed by the conclusion of this work in Section VI

\section{BACKGROUND}

\section{A. Graph Laplacian Matrices and Quadratic Forms}

Consider a weighted, undirected and connected graph $G=$ $(V, E, \omega)$, where $V$ denotes a set of vertices, $E$ denotes a set of edges, and $\omega$ denotes a weight function that assigns a positive weight to each edge. As shown in Fig. 1. the Laplacian matrix of graph $G$ can be defined as follows:

$$
\mathbf{L}_{\mathbf{G}}(p, q)= \begin{cases}-\omega(p, q) & \text { if }(p, q) \in E \\ \sum_{(p, t) \in E} \omega(p, t) & \text { if }(p=q) \\ 0 & \text { if otherwise. }\end{cases}
$$

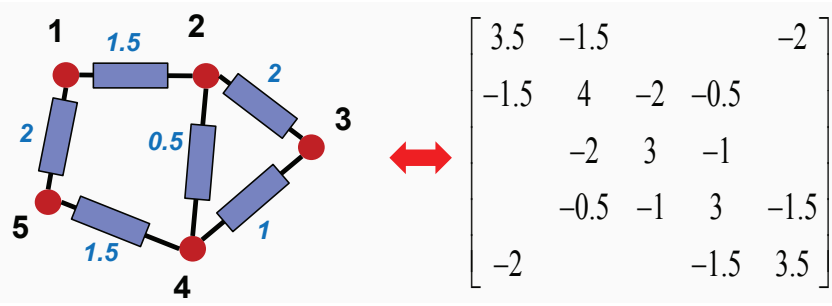

Fig. 1. A resistor network (conductance value of each element is shown) and its graph Laplacian matrix.

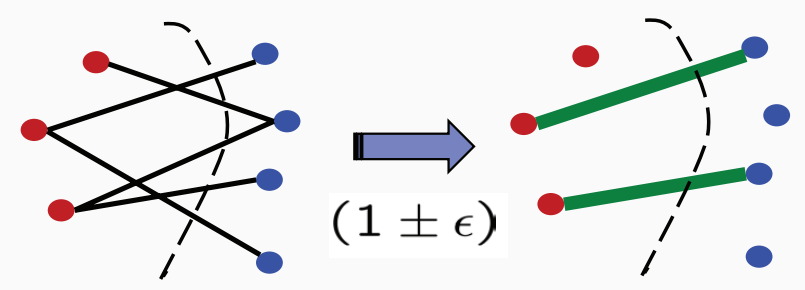

Fig. 2. Cut sparsifier preserves cuts of a graph.

It can be shown that every graph Laplacian matrix is an SDD matrix, which also can be considered as an admittance matrix of a resistor circuit network. For any real vector $\mathrm{x} \in \mathbb{R}^{V}$, the Laplacian quadratic form of graph $G$ is defined as:

$$
\mathbf{x}^{\top} \mathbf{L}_{\mathbf{G}} \mathbf{x}=\sum_{(p, q) \in E} \omega_{p, q}(x(p)-x(q))^{2},
$$

which can be considered as the Joule heat dissipated in the resistor network when $\mathrm{x}$ is a voltage vector.

\section{B. Graph Sparsification and Its Applications}

Classic graph sparsification problem can be described as follows: given a graph $G=(V, E, \omega)$ and its Laplacian matrix $\mathbf{L}_{\mathrm{G}}$, graph sparsification aims to find a subgraph (a.k.a graph sparsifier) $P=\left(V, E_{s}, \omega_{s}\right)$ and its Laplacian $\mathbf{L}_{\mathbf{P}}$ so that the sparse subgraph will retain all vertices but significantly less number of edges compared to the original graph.

Graph sparsifiers typically fall into the following two categories: the cut sparsifier [4] and spectral sparsifier [29]. The cut sparsifier preserves the values of cuts in a graph as shown in Fig. 2, whereas spectral sparsifier preserves eigenvalues and eigenvectors of the original graph. It has been shown that a good cut sparsifier may not always be a good spectral sparsifier, while the spectral sparsifier is always a stronger notion than the cut sparsifier [29].

To illustrate the role of graph sparsification in numerical computation applications, consider the following example of the standard PCG algorithm for solving SDD matrices. PCG can find an $\epsilon$-accurate solution in at most $O\left(\kappa\left(\mathbf{L}_{\mathbf{G}}, \mathbf{L}_{\mathbf{P}}\right)^{1 / 2} \log \epsilon^{-1}\right)$ iterations, where the relative condition number $\kappa\left(\mathbf{L}_{\mathbf{G}}, \mathbf{L}_{\mathbf{P}}\right)$ is defined as follows:

$$
\kappa\left(\mathbf{L}_{\mathbf{G}}, \mathbf{L}_{\mathbf{P}}\right)=\frac{\lambda_{\max }}{\lambda_{\min }}
$$



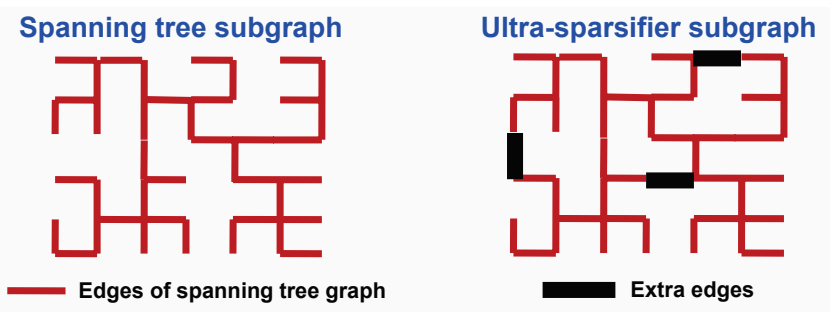

Fig. 3. A spanning tree and its ultra-sparsifier subgraph.

where $\lambda_{\min }$ and $\lambda_{\max }$ denote the smallest and largest nonzero generalized eigenvalues 1 that satisfy:

$$
\mathbf{L}_{\mathbf{G}} \mathbf{u}=\lambda \mathbf{L}_{\mathbf{P}} \mathbf{u},
$$

with $\mathbf{u}$ denoting the eigenvector corresponding to the generalized eigenvalue $\lambda$. It is desired that the preconditioner $\mathbf{L}_{\mathbf{P}}$ matrix should be very similar to $\mathbf{L}_{\mathbf{G}}$ and result in a much smaller relative condition number. It is also desired that $\mathbf{L}_{\mathbf{P}}$ should be much sparser than $\mathbf{L}_{\mathbf{G}}$ so that the overall cost of the preconditioned iterations can be much lower than directly solving the original matrix. It is obvious that a graph sparsifier with good spectral approximation of the original matrix can be immediately adopted as a preconditioner for solving SDD or SDD-like matrices [14], [15], [19], [30], [36]-[38].

\section{Spectral Graph Sparsification}

Graphs $G$ and $P$ are said to be $\sigma$-spectrally similar if for all real vectors $\mathrm{x} \in \mathbb{R}^{V}$ the following holds $|3|$ :

$$
\frac{\mathbf{x}^{\top} \mathbf{L}_{\mathbf{P}} \mathbf{x}}{\sigma} \leq \mathbf{x}^{\top} \mathbf{L}_{\mathbf{G}} \mathbf{x} \leq \sigma \mathbf{x}^{\top} \mathbf{L}_{\mathbf{P}} \mathbf{x} .
$$

It can be shown that the relative condition number $\kappa\left(\mathbf{L}_{\mathbf{G}}, \mathbf{L}_{\mathbf{P}}\right) \leq \sigma^{2}$. Note that a graph sparsifier that can result in a smaller relative condition number will also be spectrally similar to the original graph, and thus can lead to faster convergence of iterative methods, such as Krylov-subspace iterative methods. For complete graphs the Ramanujan graphs are among the best spectral graph sparsifiers [2], whereas for arbitrary graphs the linear-sized Twice-Ramanujan graphs can achieve the same spectral similarity with twice as many as the edges in Ramanujan graphs [2], [20]. However, it still remains unclear if there is a practically-efficient algorithm for constructing linear-sized spectral sparsifiers.

In this work, we define spectrally-critical edges to be the ones that can mostly perturb the graph spectral properties, such as the first few Laplacian eigenvalues and eigenvectors. Recent approaches for constructing nearly-linear-sized spectral sparsifiers aim to dramatically reduce relative condition number, which typically include the following two key steps (as shown in Fig. 3] [17], [19], [27], [30]:

1) Extract an initial spanning tree from the original graph as a backbone of the sparsifier;

\footnotetext{
${ }^{1}$ The smallest eigenvalue of a Laplacian matrix is always 0 with the corresponding all-1s eigenvector. For a disconnected graph, the number of zero eigenvalues equal to the number of disconnected components, which is equal to the algebraic multiplicity of 0 in the Laplacian.
}

2) Recover a small number of spectrally-critical off-tree edges to the spanning tree to form an ultra-sparsifier subgraph.

Note that since the smallest generalized eigenvalue $\lambda_{\min }$ of a spanning tree subgraph (without scaling) is no smaller than 1 , we always have $\lambda_{\max } \geq \kappa\left(\mathbf{L}_{\mathbf{G}}, \mathbf{L}_{\mathbf{P}}\right)=\frac{\lambda_{\max }}{\lambda_{\min }}$.

For step 1), recent theoretical computer science research results suggest that low-stretch spanning trees should be constructed since they will result in an upper bound of $\lambda_{\max }<O\left(m \log n(\log \log n)^{2}\right)$ that can immediately lead to the development of nearly-linear time algorithms for solving SDD matrices [19], where $m$ denotes the number of nonzeros and $n$ the number of equations in the matrix. Towards this goal, nearly-linear time low-stretch spanning tree algorithms based on star- and petal-decomposition methods have been proposed [1], [11].

For step 2), it requires to recover the most spectrally-critical off-tree edges to the spanning tree for constructing the ultrasparsifier, thereby drastically improving spectral approximation of the sparsifier. To this end, effective-resistance based edge sampling scheme [27] can be adopted for recovering these off-tree edges. However, calculating effective resistances (leverage scores for edge sampling) requires solving graph Laplacian multiple times (even when using the JohnsonLindenstrauss Lemma [27]) and thus can be rather expensive for very large graphs. It is also suggested to use stretch to replace effective resistance for edge sampling, which is more computationally efficient but will increase the number of edges sampled [19].

\section{Spectral Graph Sparsification Via EfFicient Spectral Perturbation Analysis}

In this paper, we introduce a practically-efficient, nearlylinear time spectral graph sparsification algorithm that can be efficiently applied to sparsify large-scale real-world graphs (Laplacian matrices). We show that for an initial spectrallycritical spanning-tree subgraph, such as a low-stretch spanning tree, the proposed algorithm can always efficiently identify the most spectrally-critical off-tree edges to be added to the spanning tree, thereby drastically reducing the relative condition number of the preconditioned system. As a result, an ultra-sparse yet spectrally-similar subgraph can be constructed and leveraged for solving sparse SDD matrices as well as other graph related problems in nearly-linear time.

\section{A. Overview of Our Approach}

The overview of the proposed method for similarity-aware spectral sparsification of undirected graphs has been summarized as follows. For a given input graph, the following key procedures are involved in the proposed algorithm flow: (a) low-stretch spanning tree [1], [11] extraction based on its original graph Laplacian; (b) spectral (generalized eigenvalue) embedding and filtering of off-tree edges by leveraging the recent spectral perturbation analysis framework [12]; (c) iterative sparsifier improvement (graph densification) by gradually adding small portions of dissimilar off-tree edges to the spanning tree.

In the rest of this paper, we assume that $G=(V, E, w)$ is a weighted, undirected and connected graph, whereas $P=$ 


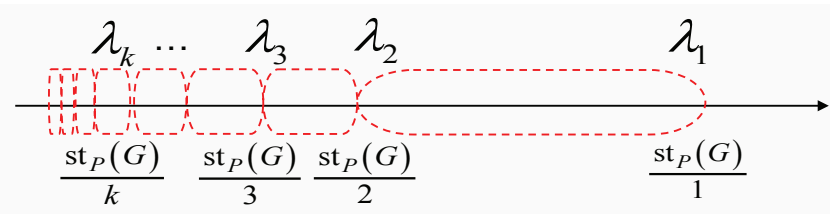

Fig. 4. A nearly worst-case distribution of generalized eigenvalues for a spanning-tree preconditioned system.

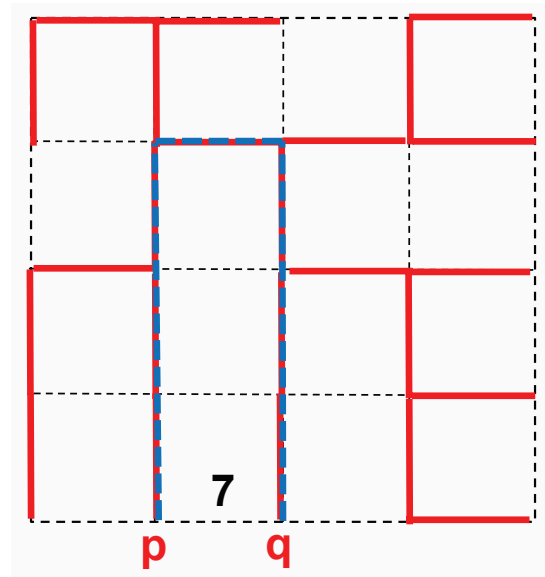

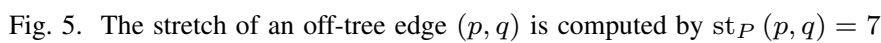
for a weighted graph with equal edge weight.

$\left(V, E_{s}, w_{s}\right)$ is its sparsifier. To simplify the our analysis, we assume the edge weights in the sparsifier remain the same as the original ones, though iterative edge weight re-scaling schemes via stochastic gradient descent (SGD) optimization [40] can be applied to further improve the spectral approximation. The descending eigenvalues of $\mathbf{L}_{\mathbf{P}}^{+} \mathbf{L}_{\mathbf{G}}$ are denoted by $\lambda_{\max }=\lambda_{1} \geq \lambda_{2} \geq \cdots \geq \lambda_{n} \geq 1$, where $\mathbf{L}_{\mathbf{P}}^{+}$denotes the Moore-Penrose pseudoinverse of $\mathbf{L}_{\mathbf{P}}$.

\section{B. Spanning Tree as A Spectral Sparsifier}

Recent work shows that when using a spanning tree subgraph as a spectral graph sparsifier or preconditioner, $\mathbf{L}_{\mathbf{P}}^{+} \mathbf{L}_{\mathbf{G}}$ will not have many large eigenvalues [31]: it has at most $k$ eigenvalues greater than $\frac{\operatorname{st}_{P}(G)}{k}$, where $\operatorname{st}_{P}(G)$ denotes the stretch of the original graph $G$ with respect to the spanning tree subgraph $P$ defined as [31]:

$$
\operatorname{st}_{P}(G)=\sum_{(p, q) \in E} \operatorname{st}_{P}(p, q),
$$

where $\operatorname{st}_{P}(p, q)$ denotes the stretch of edge $(p, q)$ defined as:

$$
\operatorname{st}_{P}(p, q)=\omega_{p, q}\left(\sum_{f \in S} \frac{1}{\omega_{f}}\right),
$$

where $S$ denotes the set of edges in the path in the spanning tree $P$ from $p$ to $q$. A nearly-worst case distribution of eigenvalues with $\lambda_{i} \leq \operatorname{st}_{P}(G) / i$ has been shown in Fig. 4
Define $\mathbf{e}_{\mathbf{p}} \in \mathbb{R}^{V}$ to be a vector with only the $p$-th element being 1 and others being 0 . Also define $\mathbf{e}_{\mathbf{p}, \mathbf{q}}=\mathbf{e}_{\mathbf{p}}-\mathbf{e}_{\mathbf{q}}$. Then the trace of $\mathbf{L}_{\mathbf{P}}^{+} \mathbf{L}_{\mathbf{G}}$ becomes [31]:

$$
\begin{aligned}
\operatorname{Tr}\left(\mathbf{L}_{\mathbf{P}}^{+} \mathbf{L}_{\mathbf{G}}\right) & =\sum_{i=1}^{n} \lambda_{i}=\sum_{(p, q) \in E} \omega_{p, q} \operatorname{Tr}\left(\mathbf{L}_{\mathbf{P}}^{+} \mathbf{e}_{\mathbf{p}, \mathbf{q}} \mathbf{e}_{\mathbf{p}, \mathbf{q}}^{\top}\right) \\
& =\sum_{(p, q) \in E} \omega_{p, q} \operatorname{Tr}\left(\mathbf{e}_{\mathbf{p}, \mathbf{q}}^{\top} \mathbf{L}_{\mathbf{P}}^{+} \mathbf{e}_{\mathbf{p}, \mathbf{q}}\right) \\
& =\sum_{(p, q) \in E} \omega_{p, q} \mathbf{e}_{\mathbf{p}, \mathbf{q}}^{\top} \mathbf{L}_{\mathbf{P}}^{+} \mathbf{e}_{\mathbf{p}, \mathbf{q}} \\
& =\operatorname{st}_{P}(G) \geq \lambda_{1}=\lambda_{\max } .
\end{aligned}
$$

The above result indicates that the total stretch $\operatorname{st}_{P}(G)$ is bounded by $\lambda_{1}$. Therefore, it is suggested that a spanning tree with low stretch should be constructed so that the preconditioned system will have a small relative condition number. It has been shown that if each of the largest eigenvalues can be fixed (dramatically decreased) by recovering a small number of off-tree edges to the spanning tree, an ultra-sparsifier with totally $n+o(n)$ edges can be created to provide a good spectral approximation of $G$ [17], [19], [30]. For instance, it has been shown that an ultra-sparsifier with a relative condition number $\alpha k^{2}$ can be constructed by adding at most $n / k$ off-tree edges to the spanning tree subgraph [30].

\section{Perturbation Analysis of Generalized Eigenvalue Problems}

Consider the following first-order eigenvalue perturbation problem:

$$
\mathbf{L}_{\mathbf{G}}\left(\mathbf{u}_{\mathbf{i}}+\delta \mathbf{u}_{\mathbf{i}}\right)=\left(\lambda_{\mathbf{i}}+\delta \lambda_{\mathbf{i}}\right)\left(\mathbf{L}_{\mathbf{P}}+\delta \mathbf{L}_{\mathbf{P}}\right)\left(\mathbf{u}_{\mathbf{i}}+\delta \mathbf{u}_{\mathbf{i}}\right),
$$

where a perturbation $\delta \mathbf{L}_{\mathbf{P}}$ is applied to $\mathbf{L}_{\mathbf{P}}$, leading to the perturbations in generalized eigenvalues and eigenvectors $\lambda_{i}+$ $\delta \lambda_{i}$ and $\mathbf{u}_{\mathbf{i}}+\delta \mathbf{u}_{\mathbf{i}}$ for $i=1, \ldots, n$, respectively. By keeping only the first-order terms, (9) becomes:

$$
\mathbf{L}_{\mathbf{G}} \delta \mathbf{u}_{\mathbf{i}}=\lambda_{\mathbf{i}} \mathbf{L}_{\mathbf{P}} \delta \mathbf{u}_{\mathbf{i}}+\delta \lambda_{\mathbf{i}} \mathbf{L}_{\mathbf{P}} \mathbf{u}_{\mathbf{i}}+\lambda_{\mathbf{i}} \delta \mathbf{L}_{\mathbf{P}} \mathbf{u}_{\mathbf{i}}
$$

Expressing $\delta \mathbf{u}_{\mathbf{i}}$ with the original eigenvectors $\mathbf{u}_{\mathbf{j}}$ leads to:

$$
\delta \mathbf{u}_{\mathbf{i}}=\sum_{\mathbf{j}=\mathbf{1}}^{\mathbf{n}} \zeta_{\mathbf{i j}} \mathbf{u}_{\mathbf{j}}
$$

where $\mathbf{u}_{\mathbf{j}}$ can always be constructed to satisfy:

$$
\mathbf{u}_{\mathbf{i}}^{\top} \mathbf{L}_{\mathbf{P}} \mathbf{u}_{\mathbf{j}}=\left\{\begin{array}{l}
1, i=j \\
0, i \neq j .
\end{array}\right.
$$

Substituting (11) into 10 leads to:

$$
\begin{aligned}
& \sum_{j=1}^{n} \zeta_{\mathbf{i j}} \lambda_{\mathbf{j}} \mathbf{L}_{\mathbf{P}} \mathbf{u}_{\mathbf{j}} \\
& =\lambda_{i} \mathbf{L}_{\mathbf{P}}\left(\sum_{\mathbf{j}=\mathbf{1}}^{\mathbf{n}} \zeta_{\mathbf{i j}} \mathbf{u}_{\mathbf{j}}\right)+\delta \lambda_{\mathbf{i}} \mathbf{L}_{\mathbf{P}} \mathbf{u}_{\mathbf{i}}+\lambda_{\mathbf{i}} \delta \mathbf{L}_{\mathbf{P}} \mathbf{u}_{\mathbf{i}}
\end{aligned}
$$

Multiplying $u_{i}^{\top}$ to both sides of 13 results in:

$$
\begin{aligned}
& \mathbf{u}_{\mathbf{i}}^{\top}\left(\sum_{\mathbf{j}=\mathbf{1}}^{\mathbf{n}} \zeta_{\mathbf{i} \mathbf{j}} \lambda_{\mathbf{j}} \mathbf{L}_{\mathbf{P}} \mathbf{u}_{\mathbf{j}}\right)= \\
& \lambda_{\mathbf{i}} \mathbf{u}_{\mathbf{i}}^{\top} \mathbf{L}_{\mathbf{P}}\left(\sum_{\mathbf{j}=\mathbf{1}}^{\mathbf{n}} \zeta_{\mathbf{i} \mathbf{j}} \mathbf{u}_{\mathbf{j}}\right)+\delta \lambda_{\mathbf{i}} \mathbf{u}_{\mathbf{i}}^{\top} \mathbf{L}_{\mathbf{P}} \mathbf{u}_{\mathbf{i}}+\lambda_{\mathbf{i}} \mathbf{u}_{\mathbf{i}}^{\top} \delta \mathbf{L}_{\mathbf{P}} \mathbf{u}_{\mathbf{i}},
\end{aligned}
$$


which immediately leads to:

$$
\delta \lambda_{\mathbf{i}}=-\lambda_{\mathbf{i}} \frac{\mathbf{u}_{\mathbf{i}}^{\top} \delta \mathbf{L}_{\mathbf{P}} \mathbf{u}_{\mathbf{i}}}{\mathbf{u}_{\mathbf{i}}^{\top} \mathbf{L}_{\mathbf{P}} \mathbf{u}_{\mathbf{i}}}=-\lambda_{\mathbf{i}} \mathbf{u}_{\mathbf{i}}^{\top} \delta \mathbf{L}_{\mathbf{P}} \mathbf{u}_{\mathbf{i}}
$$

Expanding $\delta \mathbf{L}_{\mathbf{P}}$ that includes multiple extra off-tree edges $(p, q)$ leads to:

$$
\delta \mathbf{L}_{\mathbf{P}}=\sum_{(\mathbf{p}, \mathbf{q}) \in \mathbf{E} \backslash \mathbf{E}_{\mathbf{s}}} \omega_{\mathbf{p}, \mathbf{q}} \mathbf{e}_{\mathbf{p}, \mathbf{q}} \mathbf{e}_{\mathbf{p}, \mathbf{q}}^{\top}
$$

The above leads to the following based on 15 :

$$
\delta \lambda_{\mathbf{i}}=-\lambda_{\mathbf{i}} \sum_{(\mathbf{p}, \mathbf{q}) \in \mathbf{E} \backslash \mathbf{E}_{\mathbf{s}}} \mathbf{w}_{\mathbf{p}, \mathbf{q}} \mathbf{u}_{\mathbf{i}}^{\top} \mathbf{e}_{\mathbf{p}, \mathbf{q}} \mathbf{e}_{\mathbf{p}, \mathbf{q}}^{\top} \mathbf{u}_{\mathbf{i}}
$$

It is obvious from (17) that the reduction of $\lambda_{i}$ is proportional to the Joule heat produced by the extra off-tree edges when its unperturbed eigenvector $\mathbf{u}_{\mathbf{i}}$ is applied as a nodevoltage vector. For instance, the voltage difference between nodes $p$ and $q$ is computed by

$$
v_{p, q}=\mathbf{u}_{\mathbf{i}}^{\top} \mathbf{e}_{\mathbf{p}, \mathbf{q}},
$$

which results in the following Joule heat for the off-tree edge $(p, q)$ :

$$
h_{p, q}=\omega_{p, q} v_{p, q}^{2} .
$$

Therefore, the perturbation of eigenvalue $\lambda_{i}$ becomes:

$$
\delta \lambda_{i}=-\lambda_{i} \sum_{(p, q) \in E \backslash E_{s}} h_{p, q} .
$$

Consequently, adding the off-tree edges with the largest Joule heat values computed using the dominant generalized eigenvector $\left(\mathbf{u}_{\mathbf{1}}\right)$ that corresponds to the largest eigenvalue $\left(\lambda_{1}\right)$ will dramatically reduce the relative condition number thereby improving the spectral approximation of the subgraph. Repeating the above procedures for all large eigenvalues will lead to very good spectral graph sparsifiers.

\section{Why Dominant Generalized Eigenvectors?}

As shown in the previous perturbation analysis, dominant generalized eigenvectors can help identify the most spectrallycritical off-tree edges. Alternatively, the following CourantFischer theorem can be leveraged for better understanding why generalized eigenvectors should be used for spectral sparsification tasks. Assigning each node in the graph with an integer value either 0 or 1 , the corresponding Laplacian quadratic form measures the boundary size (cut) of a node set. For example, if a node set $Q$ is defined as

$$
Q \stackrel{\text { def }}{=}\{q \in V: x(q)=1\},
$$

then the number of edges going out of $Q$ becomes:

$$
\mathbf{x}^{\top} \mathbf{L}_{G} \mathbf{x}=\operatorname{cut}(Q, \bar{Q})=\left|\partial_{G}(Q)\right|,
$$

where the boundary of $Q$ in $G$ is defined as

$$
\partial_{G}(Q) \stackrel{\text { def }}{=}\{(p, q) \in E: p \notin Q, q \in Q\} .
$$

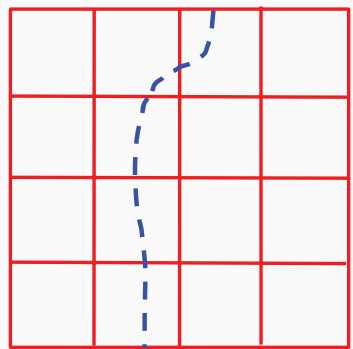

Graph G

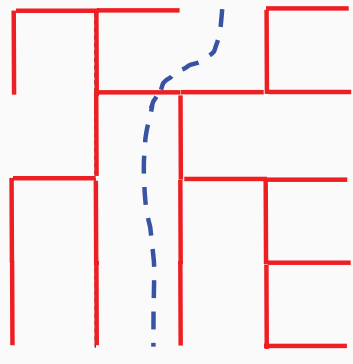

Subgraph P
Courant - Fischer theorem for generalized eigenvalues

$$
\lambda_{\max }=\max _{\|x\|=1} \frac{x^{T} L_{G} x}{x^{T} L_{P} x} \Rightarrow \frac{\text { max cuts in } \mathrm{G}}{\text { min cuts in } \mathrm{P}} \Rightarrow \text { max mismatch in } \mathrm{P}
$$

Fig. 6. An alternative view based on Courant-Fischer theorem.

The Courant-Fischer theorem for generalized eigenvalue problems allows finding dominant eigenvalues and eigenvectors by solving the following optimization task:

$$
\lambda_{\max }=\max _{\substack{|\mathbf{x}| \neq 0 \\ \mathbf{x}^{\top} \mathbf{1}=0}} \frac{\mathbf{x}^{\top} \mathbf{L}_{G} \mathbf{x}}{\mathbf{x}^{\top} \mathbf{L}_{P} \mathbf{x}} \geq \max _{\substack{|\mathbf{x}| \neq 0 \\ x(p) \in\{0,1\}}} \frac{\mathbf{x}^{\top} \mathbf{L}_{G} \mathbf{x}}{\mathbf{x}^{\top} \mathbf{L}_{P} \mathbf{x}}=\max \frac{\left|\partial_{G}(Q)\right|}{\left|\partial_{P}(Q)\right|},
$$

where $\mathbf{1} \in \mathbb{R}^{V}$ is the all-one vector, and the boundary of $Q$ in $P$ is defined as

$$
\partial_{P}(Q) \stackrel{\text { def }}{=}\left\{(p, q) \in E_{s}: p \notin Q, q \in Q\right\},
$$

which leads to the following equations:

$$
\mathbf{x}^{\top} \mathbf{L}_{G} \mathbf{x}=\left|\partial_{G}(Q)\right|, \mathbf{x}^{\top} \mathbf{L}_{P} \mathbf{x}=\left|\partial_{P}(Q)\right| .
$$

Then (24) indicates that finding the dominant generalized eigenvector would be closely related to finding $Q$ such that $\frac{\left|\partial_{G}(Q)\right|}{\partial_{P}(Q) \mid}$ or the ratio of the boundary sizes in the original graph $G$ and subgraph $P$ is maximized. As a result, $\lambda_{\max }=\lambda_{1}$ becomes the upper bound of the mismatch in boundary (cut) size between $G$ and $P$, as shown in Fig. 6

\section{E. Problem Formulation}

Once $Q$ or $\partial_{G}(Q)$ is found using dominant generalized eigenvectors, we can recover a small number of edges from $\partial_{G}(Q)$ to $P$ in order to dramatically reduce the maximum mismatch $\left(\lambda_{1}\right)$, and thus improve the spectral approximation of $P$. To this end, we propose the following problem formulation for spectral graph sparsification:

$$
\min _{\mathbf{L}_{P}}\left\{\max _{\mathbf{x}}\left(\frac{\mathbf{x}^{\top} \mathbf{L}_{G} \mathbf{x}}{\mathbf{x}^{\top} \mathbf{L}_{P} \mathbf{x}}\right)+\beta\left\|\mathbf{L}_{P}\right\|_{1}\right\},
$$

where $\mathbf{L}_{P}$ denotes Laplacian matrix of the subgraph $P, \mathbf{x}^{\top} \mathbf{1}=$ 0 and $|\mathbf{x}| \neq 0$. 27) aims to minimize the largest generalized eigenvalue by adding the minimum amount of edges into the subgraph $P$, which can be solved iteratively by repeating the following two steps: 1) compute the generalized eigenvector corresponding to the largest (dominant) eigenvalue, and 2) identify the most spectrally-critical off-tree edges that can mostly decrease the dominant eigenvalue(s) and add them into 
the subgraph $P$. Once the dominant eigenvalue is small enough (e.g., $\lambda=10), P$ will be highly spectrally similar to the original graph $G$.

However, computing the dominant eigenvalue and its eigenvector can sometimes be too costly for large-scale graph Laplacian matrices, even when state-of-the-art eigenvalue decomposition methods are adopted [25]. Additionally, there can still be too many eigenvalues to be fixed in order to achieve a desired spectral similarity level (relative condition number).

\section{F. Edge Embedding with Approximate Dominant Eigenvector}

To more efficiently identify critical off-tree edges, approximate dominant generalized eigenvectors and eigenvalues of (4) can be exploited. To this end, a generalized power iteration procedure that can be computed in nearly-linear time has been proposed in [12]. In the rest of this paper, we assume that a very small positive diagonal element is added to a randomly selected row of the graph Laplacian matrix to convert the Laplacian matrix into a full-rank matrix. We express an initial random vector $\mathbf{h}_{\mathbf{0}}$ that is orthogonal to the all-one vector using generalized eigenvectors $\mathbf{u}_{\mathbf{i}}$ as follows:

$$
\mathbf{h}_{\mathbf{0}}=\sum_{\mathbf{i}=\mathbf{1}}^{\mathbf{n}} \alpha_{\mathbf{i}} \mathbf{u}_{\mathbf{i}}
$$

where $\mathbf{1}^{\top} \mathbf{h}_{\mathbf{0}}=0$. Applying $t$-step power iterations to the generalized eigenvalue problem, we have

$$
\mathbf{h}_{\mathbf{t}}=\left(\mathbf{L}_{\mathbf{P}}^{-\mathbf{1}} \mathbf{L}_{\mathbf{G}}\right)^{\mathbf{t}} \mathbf{h}_{\mathbf{0}}=\sum_{\mathbf{i}=\mathbf{1}}^{\mathbf{n}} \alpha_{\mathbf{i}} \lambda_{\mathbf{i}}^{\mathbf{t}} \mathbf{u}_{\mathbf{i}}
$$

The Laplacian quadratic form function $Q_{\delta \mathbf{L}_{\mathbf{P}}}\left(\mathbf{h}_{\mathbf{t}}\right)=$ $\mathbf{h}_{\mathbf{t}}^{\top} \delta \mathbf{L}_{\mathbf{P}} \mathbf{h}_{\mathbf{t}}$ becomes:

$$
\begin{aligned}
& Q_{\delta L_{P}}\left(\mathbf{h}_{\mathbf{t}}\right)=\mathbf{h}_{\mathbf{t}}^{\top} \delta \mathbf{L}_{\mathbf{P}} \mathbf{h}_{\mathbf{t}}=\left(\sum_{i=1}^{n} \alpha_{i} \lambda_{i}^{t} \mathbf{u}_{\mathbf{i}}\right)^{\top} \delta \mathbf{L}_{\mathbf{P}}\left(\sum_{j=1}^{n} \alpha_{j} \lambda_{j}^{t} \mathbf{u}_{\mathbf{j}}\right) \\
& =\sum_{\mathbf{i}=\mathbf{1}}^{\mathbf{n}}\left(\alpha_{\mathbf{i}} \lambda_{\mathbf{i}}^{\mathbf{t}}\right)^{\mathbf{2}} \mathbf{u}_{\mathbf{i}}^{\top} \delta \mathbf{L}_{\mathbf{P}} \mathbf{u}_{\mathbf{i}}+\sum_{\mathbf{i}=\mathbf{1} \mathbf{j}=\mathbf{1}, \mathbf{j} \neq \mathbf{i}}^{\mathbf{n}} \alpha_{\mathbf{i}} \alpha_{\mathbf{j}} \lambda_{\mathbf{i}}^{\mathbf{t}} \lambda_{\mathbf{j}}^{\mathbf{t}} \mathbf{u}_{\mathbf{j}}^{\top} \delta \mathbf{L}_{\mathbf{P}} \mathbf{u}_{\mathbf{i}} .
\end{aligned}
$$

Substituting 16 into 30, $Q_{\delta \mathbf{L}_{\mathbf{P}}}\left(\mathbf{h}_{\mathbf{t}}\right)$ becomes:

$$
\begin{aligned}
Q_{\delta L_{P}}\left(\mathbf{h}_{\mathbf{t}}\right) & =\sum_{(p, q) \in E \backslash E_{s}} \omega_{p, q}\left(\sum_{i=1}^{n} \alpha_{i} \lambda_{i}^{t} \mathbf{u}_{\mathbf{i}}^{\top} \mathbf{e}_{\mathbf{p}, \mathbf{q}}\right)^{2} \\
& =\sum_{(p, q) \in E \backslash E_{s}} \omega_{p, q} \bar{v}_{p, q}^{2},
\end{aligned}
$$

which indicates that an off-tree edge with greater $\left|\bar{v}_{p, q}\right|$ or Joule heat $\omega_{p, q} \bar{v}_{p, q}^{2}$ for $t>0$ will be a more spectrally-critical off-tree edge that can significantly influence large generalized eigenvalues. Expanding (31) leads to:

$$
\begin{aligned}
& Q_{\delta L_{P}}\left(\mathbf{h}_{\mathbf{t}}\right)=\sum_{i=1}^{n} \alpha_{i}^{2} \lambda_{i}^{2 t} \sum_{(p, q) \in E \backslash E_{s}} \omega_{p, q}\left(\mathbf{u}_{\mathbf{i}}^{\top} \mathbf{e}_{\mathbf{p}, \mathbf{q}}\right)^{\mathbf{2}}+ \\
& \sum_{i=1}^{n} \sum_{j=1, j \neq i}^{n} \alpha_{i} \alpha_{j} \lambda_{i}^{t} \lambda_{j}^{t} \sum_{(p, q) \in E \backslash E_{s}} \omega_{p, q} \mathbf{u}_{\mathbf{i}}^{\top} \mathbf{e}_{\mathbf{p}, \mathbf{q}} \mathbf{u}_{\mathbf{j}}^{\top} \mathbf{e}_{\mathbf{p}, \mathbf{q}}
\end{aligned}
$$

If we further define:

$$
\delta \mathbf{L}_{\mathbf{P}, \max }=\mathbf{L}_{\mathbf{G}}-\mathbf{L}_{\mathbf{P}}
$$

which can be considered as an extreme-case Laplacian matrix that includes all off-tree edges that belong to the original graph $G$ but not the subgraph $P$, then the following equation holds:

$$
\begin{aligned}
& \sum_{i=1}^{n} \sum_{j=1, j \neq i}^{n} \alpha_{i} \alpha_{j} \lambda_{i}^{t} \lambda_{j}^{t} \sum_{(p, q) \in E \backslash E_{s}} w_{p, q} \mathbf{u}_{\mathbf{i}}^{\top} \mathbf{e}_{\mathbf{p}, \mathbf{q}} \mathbf{u}_{\mathbf{j}}^{\top} \mathbf{e}_{\mathbf{p}, \mathbf{q}}= \\
& \sum_{i=1}^{n} \sum_{j=1, j \neq i}^{n} \alpha_{i} \alpha_{j} \lambda_{i}^{t} \lambda_{j}^{t} \mathbf{u}_{\mathbf{i}}^{\top}\left(\mathbf{L}_{\mathbf{G}}-\mathbf{L}_{\mathbf{P}}\right) \mathbf{u}_{\mathbf{j}}=\mathbf{0}
\end{aligned}
$$

which leads to the edge-based expansion of the quadratic form for $\delta \mathbf{L}_{\mathbf{P}, \max }$ as follows:

$$
\begin{aligned}
& Q_{\delta \mathbf{L}_{\mathbf{P}, \max }}\left(\mathbf{h}_{\mathbf{t}}\right)=\mathbf{h}_{\mathbf{t}}^{\top} \delta \mathbf{L}_{\mathbf{P}, \mathbf{m a x}} \mathbf{h}_{\mathbf{t}}=\sum_{\mathbf{i}=\mathbf{1}}^{\mathbf{n}}\left(\alpha_{\mathbf{i}} \lambda_{\mathbf{i}}^{\mathbf{t}}\right)^{\mathbf{2}}\left(\lambda_{\mathbf{i}}-\mathbf{1}\right) \\
& =\sum_{(p, q) \in E \backslash E_{s}} w_{p, q} \sum_{i=1}^{n} \alpha_{i}^{2} \lambda_{i}^{2 t}\left(\mathbf{u}_{\mathbf{i}}^{\top} \mathbf{e}_{\mathbf{p}, \mathbf{q}}\right)^{\mathbf{2}} .
\end{aligned}
$$

It is obvious that (31), or 35) will allow ranking each offtree edge according to its spectral-criticality level. (35) also indicates that adding all off-tree edges with nonzero Joule heat values back to $P$ will immediately bring all generalized eigenvalues to 1 . It should be noted that the required number of generalized power iterations can be rather small (e.g. $t=2$ ) in practice to observe good result.

\section{G. Spectrally-unique Off-tree Edges}

Define a spectrally-unique off-tree edge $\left(p_{i}, q_{i}\right)$ to be the offtree edge that can completely and only fix one large (dominant) generalized eigenvalue $\lambda_{i}$, though each edge will usually influence more than one eigenvalues and eigenvectors according to (35). Then the following truncated expansion of the Laplacian quadratic form can be obtained when considering the top $k$ most dominant yet spectrally-unique off-tree edges for fixing the top $k$ largest eigenvalues:

$$
\begin{aligned}
Q_{\delta L_{P, \text { max }}}\left(\mathbf{h}_{\mathbf{t}}\right) & \approx \sum_{i=1}^{k} w_{p_{i}, q_{i}} \alpha_{i}^{2} \lambda_{i}^{2 t}\left(u_{i}^{\top} e_{p_{i}, q_{i}}\right)^{2} \\
& =\sum_{i=1}^{k} \alpha_{i}^{2} \lambda_{i}^{2 t}\left(\lambda_{i}-1\right),
\end{aligned}
$$

where we should be able to find $\gamma_{i} \neq 0$ such that:

$$
\mathbf{e}_{\mathbf{p}_{\mathbf{i}}, \mathbf{q}_{\mathbf{i}}}=\gamma_{\mathbf{i}} \mathbf{L}_{\mathbf{P}} \mathbf{u}_{\mathbf{i}}
$$

while the following will also be satisfied:

$$
\mathbf{u}_{\mathbf{j}}^{\top} \mathbf{e}_{\mathbf{p}_{\mathbf{i}}, \mathbf{q}_{\mathbf{i}}}=\left\{\begin{array}{l}
\gamma_{i}, i=j, \\
0, i \neq j .
\end{array}\right.
$$

Obviously, for random coefficients $\alpha_{i}$, the following must be satisfied for a spectrally-unique off-tree edge:

$$
w_{p_{i}, q_{i}} \gamma_{i}^{2}=\lambda_{i}-1
$$

Consequently, if there is an off-tree edge $\left(p_{i}, q_{i}\right)$ with weight $w_{p_{i}, q_{i}}$ that satisfies:

$$
\mathbf{e}_{\mathbf{p}_{\mathbf{i}}}-\mathbf{e}_{\mathbf{q}_{\mathbf{i}}}= \pm \sqrt{\frac{\lambda_{i}-1}{w_{p_{i}, q_{i}}}} \mathbf{L}_{\mathbf{P}} \mathbf{u}_{\mathbf{i}}
$$

adding this off-tree edge back to the spanning tree will completely fix the corresponding eigenvalue $\lambda_{i}$. Then the effective resistance of edge $\left(p_{i}, q_{i}\right)$ in $P$ becomes:

$$
R_{e_{i}}^{\mathbf{e f f}}=\mathbf{e}_{p_{i}, q_{i}}^{\top} \mathbf{L}_{\mathbf{P}}^{+} \mathbf{e}_{p_{i}, q_{i}}=\gamma_{i}^{2} \mathbf{u}_{\mathbf{i}}^{\top} \mathbf{L}_{\mathbf{P}} \mathbf{u}_{\mathbf{i}}=\gamma_{i}^{2},
$$


which immediately leads to:

$$
Q_{\delta \mathbf{L}_{\mathbf{P}, \max }}\left(\mathbf{h}_{\mathbf{t}}\right) \approx \sum_{i=1}^{k} \alpha_{i}^{2} \lambda_{i}^{2 t} w_{p_{i}, q_{i}} R_{e_{i}}^{\mathrm{eff}} \approx \sum_{i=1}^{k} \alpha_{i}^{2} \lambda_{i}^{2 t+1} .
$$

Since the stretch of off-tree edge $\left(p_{i}, q_{i}\right)$ is computed by $\operatorname{st}_{P}\left(p_{i}, q_{i}\right)=w_{p_{i}, q_{i}} R_{e_{i}}^{\text {eff }}, 42$ also indicates that $\operatorname{st}_{P}\left(e_{i}\right) \approx$ $\lambda_{i}$ holds for spectrally-unique off-tree edges. Consequently, the key off-tree edges identified by (35) or (42) will have the largest stretch values and therefore most significantly impact the largest eigenvalues of $\mathbf{L}_{\mathbf{P}}^{+} \mathbf{L}_{\mathbf{G}}$. $\sqrt{42}$ also can be considered as a randomized version of $\operatorname{Trace}\left(\mathbf{L}_{\mathbf{P}}^{+} \mathbf{L}_{\mathbf{G}}\right)$ that is further scaled up by a factor of $\lambda_{i}^{2 t}$.

\section{H. Rank-One Update with A Spectrally-Unique Edge}

The updated generalized eigenvalue $\lambda_{i}^{\prime}$ after adding one spectrally-unique off-tree edge $\left(p_{i}, q_{i}\right)$ with weight $w_{p_{i}, q_{i}}$ back to the spanning tree for fixing eigenvalue $\lambda_{i}$ can be derived based on the Sherman-Morrison Formula and Matrix Determinant Lemma. Define matrix $A_{P}$ to be:

$$
\mathbf{A}_{\mathbf{P}}=\mathbf{L}_{\mathbf{G}}^{\frac{1}{2}} \mathbf{L}_{\mathbf{P}}^{-1} \mathbf{L}_{\mathbf{G}}^{\frac{1}{2}}
$$

which has the same set of eigenvalues of matrix $\mathbf{L}_{\mathbf{P}}^{-1} \mathbf{L}_{\mathrm{G}}$. After adding the off-tree edge $\left(p_{i}, q_{i}\right)$, the updated $\mathbf{A}_{\mathbf{P}}$ is denoted by $\mathbf{A}_{\mathbf{P}}^{\prime}$ that is expressed as:

$$
\begin{aligned}
& \mathbf{A}_{\mathbf{P}}^{\prime}=\mathbf{L}_{\mathbf{G}}^{\frac{1}{2}}\left(\mathbf{L}_{\mathbf{P}}+w_{p_{i}, q_{i}} \mathbf{e}_{\mathbf{p}_{\mathbf{i}}, \mathbf{q}_{\mathbf{i}}} \mathbf{e}_{\mathbf{p}_{\mathbf{i}}, \mathbf{q}_{\mathbf{i}}^{\top}}^{\top}\right)^{-1} \mathbf{L}_{\mathbf{G}}^{\frac{1}{2}} \\
& =\mathbf{A}_{\mathbf{P}}-\frac{w_{p_{i}, q_{i}} \mathbf{L}_{\mathrm{G}}^{\frac{1}{2}} \mathbf{L}_{\mathbf{P}}^{-1} \mathbf{e}_{\mathbf{p}_{\mathbf{i}}, \mathbf{q}_{\mathbf{i}}} \mathbf{e}_{\mathbf{p}_{1}, \mathbf{q}_{\mathbf{i}}}^{\top} \mathbf{L}_{\mathbf{P}}^{-1} \mathbf{L}_{\mathrm{G}}^{\frac{1}{2}}}{1+w_{p_{i}, q_{i}} \mathbf{e}_{\mathbf{p}_{\mathbf{i}}, \mathbf{q}_{\mathbf{i}}}^{\top} \mathbf{L}_{\mathbf{P}}^{-1} \mathbf{e}_{\mathbf{p}_{\mathbf{i}}, \mathbf{q}_{\mathbf{i}}}}=\mathbf{A}_{\mathbf{P}}-\mathbf{v}_{\mathbf{P}} \mathbf{V}_{\mathbf{P}}^{\top} \text {, }
\end{aligned}
$$

where $\mathbf{v}_{\mathbf{P}}$ is defined as:

$$
\mathbf{v}_{\mathbf{P}}=\frac{\sqrt{w_{p_{i}, q_{i}}} \mathbf{L}_{\mathbf{G}}^{\frac{1}{2}} \mathbf{L}_{\mathbf{P}}^{-1} \mathbf{e}_{\mathbf{p}_{\mathbf{i}}, \mathbf{q}_{\mathbf{i}}}}{\sqrt{1+w_{p_{i}, q_{i}} \mathbf{e}_{\mathbf{p}_{\mathbf{i}}, \mathbf{q}_{\mathbf{i}}}^{\top} \mathbf{L}_{\mathbf{P}}^{-1} \mathbf{e}_{\mathbf{p}_{\mathbf{i}}, \mathbf{q}_{\mathbf{i}}}}}=\frac{\gamma_{i} \sqrt{w_{p_{i}, q_{i}}} \mathbf{L}_{\mathbf{G}}^{\frac{1}{2}} \mathbf{u}_{\mathbf{i}}}{\sqrt{1+w_{p_{i}, q_{i}} \gamma_{i}^{2}}} .
$$

The characteristic polynomial of $\mathbf{A}_{\mathbf{P}}^{\prime}=\mathbf{A}_{\mathbf{P}}-\mathbf{v}_{\mathbf{P}} \mathbf{v}_{\mathbf{P}}^{\top}$ can be computed as follows:

$$
\begin{aligned}
& p_{\mathbf{A}_{\mathbf{P}}^{\prime}}(x)=p_{\mathbf{A}_{\mathbf{P}}-\mathbf{v}_{\mathbf{P}} \mathbf{v}_{\mathbf{P}}^{\top}}(x)=\operatorname{det}\left(\mathbf{x I}-\mathbf{A}_{\mathbf{P}}+\mathbf{v}_{\mathbf{P}} \mathbf{v}_{\mathbf{P}}^{\top}\right) \\
& =\operatorname{det}\left(\mathbf{x I}-\mathbf{A}_{\mathbf{P}}\right) \operatorname{det}\left(\mathbf{I}+\left(\mathbf{x I}-\mathbf{A}_{\mathbf{P}}\right)^{-\mathbf{1}} \mathbf{v}_{\mathbf{P}} \mathbf{v}_{\mathbf{P}}^{\top}\right) .
\end{aligned}
$$

According to Matrix Determinant Lemma, we have:

$$
p_{\mathbf{A}_{\mathbf{P}}^{\prime}}(x)=p_{\mathbf{A}_{\mathbf{P}}}(x)\left(1+\mathbf{v}_{\mathbf{P}}^{\top}\left(\mathbf{x I}-\mathbf{A}_{\mathbf{P}}\right)^{-\mathbf{1}} \mathbf{v}_{\mathbf{P}}\right) .
$$

Denoting $\mathbf{z}_{\mathbf{i}}$ for $i=1, \ldots, n$ the unit-length orthonormalized eigenvectors of matrix $\mathbf{A}_{\mathbf{P}}$ that correspond to eigenvalues $\lambda_{i}$ respectively, we have:

$$
\left(\mathbf{x} \mathbf{I}-\mathbf{A}_{\mathbf{P}}\right)^{-1}=\left(\sum_{i=1}^{n}\left(x-\lambda_{i}\right) \mathbf{z}_{\mathbf{i}} \mathbf{z}_{\mathbf{i}}^{\top}\right)^{-1}=\sum_{i=1}^{n} \frac{\mathbf{z}_{\mathbf{i}} \mathbf{z}_{\mathbf{i}}^{\top}}{x-\lambda_{i}},
$$

which leads to:

$$
p_{\mathbf{A}_{\mathbf{P}}^{\prime}}(x)=p_{\mathbf{A}_{\mathbf{P}}}(x)\left(1+\sum_{i=1}^{n} \frac{\left(\mathbf{v}_{\mathbf{P}}^{\top} \mathbf{z}_{\mathbf{i}}\right)^{2}}{x-\lambda_{i}}\right) .
$$

It has been shown in [2] that 490 indicates: 1) the latest eigenvalues after rank-one update will always be reduced if $\left.\left(\mathbf{v}_{\mathbf{P}}^{\top} \mathbf{z}_{\mathbf{i}}\right)^{2}>0 ; 2\right)$ the greater value of $\left(\mathbf{v}_{\mathbf{P}}^{\top} \mathbf{z}_{\mathbf{i}}\right)^{2}$ will result in greater reduction in $\lambda_{i}$. Therefore, if an off-tree edge satisfies
$\left(\mathbf{v}_{\mathbf{P}}^{\top} \mathbf{Z}_{\mathbf{i}}\right)^{2}>>0$ for multiple eigenvectors $\mathbf{z}_{\mathbf{i}}$, adding this edge back to the spanning tree subgraph will substantially reduce multiple eigenvalues at the same time; on the other hand, a spectrally-unique off-tree edge will substantially reduce only one eigenvalue. It can be shown that:

$$
\mathbf{L}_{\mathbf{G}}^{\frac{1}{2}} \mathbf{L}_{\mathbf{P}}^{-1} \mathbf{L}_{\mathbf{G}}^{\frac{1}{2}}\left(\frac{\mathbf{L}_{\mathbf{G}}^{\frac{1}{2}} \mathbf{u}_{\mathbf{i}}}{\left\|\mathbf{L}_{\mathbf{G}}^{\frac{1}{2}} \mathbf{u}_{\mathbf{i}}\right\|}\right)=\lambda_{\mathbf{i}}\left(\frac{\mathbf{L}_{\mathbf{G}}^{\frac{1}{2}} \mathbf{u}_{\mathbf{i}}}{\left\|\mathbf{L}_{\mathbf{G}}^{\frac{1}{2}} \mathbf{u}_{\mathbf{i}}\right\|}\right)=\lambda_{\mathbf{i}} \mathbf{z}_{\mathbf{i}},
$$

which indicates that $\mathbf{v}_{\mathbf{P}}$ is an eigenvector of matrix $\mathbf{A}_{\mathbf{P}}$ corresponding to the eigenvalue $\lambda_{i}$. Consequently, the characteristic polynomial of $\mathbf{A}_{\mathbf{P}}^{\prime}=\mathbf{A}_{\mathbf{P}}-\mathbf{v}_{\mathbf{P}} \mathbf{v}_{\mathbf{P}}^{\top}$ can be further simplified into the following form:

$p_{\mathbf{A}_{\mathbf{P}}^{\prime}}(x)=p_{\mathbf{A}_{\mathbf{P}}}(x)\left(1+\frac{\left(\mathbf{v}_{\mathbf{P}}^{\top} \mathbf{z}_{\mathbf{i}}\right)^{2}}{x-\lambda_{i}}\right)=p_{\mathbf{A}_{\mathbf{P}}}(x)\left(1+\frac{\mathbf{v}_{\mathbf{P}}^{\top} \mathbf{v}_{\mathbf{P}}}{x-\lambda_{i}}\right)$.

Therefore, the updated eigenvalue $\lambda_{i}^{\prime}$ after adding the off-tree edge can be computed by solving $p_{A_{P}^{\prime}}(x)=0$, which leads to:

$$
\lambda_{i}^{\prime}=\lambda_{i}-\mathbf{v}_{\mathbf{P}}^{\top} \mathbf{v}_{\mathbf{P}}=\frac{\lambda_{i}}{1+w_{p_{i}, q_{i}} \gamma_{i}^{2}} .
$$

For achieving the desired $\lambda_{i}^{\prime}$ after adding the off-tree edge, the edge weight should be set as:

$$
w_{p_{i}, q_{i}}=\frac{\lambda_{i}-\lambda_{i}^{\prime}}{\lambda_{i}^{\prime} \gamma_{i}^{2}} .
$$

It can be shown that when the desired $\lambda_{i}^{\prime}=1$, we have $w_{p_{i}, q_{i}}=\frac{\lambda_{i}-1}{\gamma_{i}^{2}}$, which is equivalent to 39. As a result, considering the nearly-worst case eigenvalue distribution $\lambda_{i} \leq$ $\operatorname{st}_{P}(G) / i$ shown in Fig. 4, a $\sigma$-similar spectral sparsifier with $n-1+O\left(\frac{m \log n \log \log n}{\sigma^{2}}\right)$ edges can be obtained in $O(m)$ time using the proposed method when an initial low-stretch spanning tree is given.

\section{Algorithm Flow and Complexity}

The detailed algorithm flow of the proposed spectral graph sparsification approach has been summarized as follows:

1) Extract a spanning tree subgraph (e.g. a scaled lowstretch spanning tree [1], [11]) from the original graph;

2) Perform $t$-step generalized power iterations to compute $h_{t}$ with an initial random vector;

3) Compute the spectral criticality of each edge based on the Laplacian quadratic form of $\delta L_{P, \max }$ using (35);

4) Rank each edge using its spectral criticality levels;

5) Add a small portion of dissimilar off-tree edges back to the spanning tree to form the ultra-sparse spectral graph sparsifier.

It should be noted that the proposed spectral graph sparsification approach allows ranking all off-tree edges according to their spectral criticality levels in a very efficient and effective way. Compared to the state-of-the-art sampling-based approaches that rely on effective resistance calculations, the proposed method can achieve the very similar goal of ranking spectrally critical off-tree edges.

The complexity of the proposed spectral perturbation based approach can be analyzed by considering two key steps: (a) spanning tree construction based on the original graph, and (b) ultra-sparsifier construction based on the spanning tree. Recent research has shown that low-stretch spanning trees in (a) can 


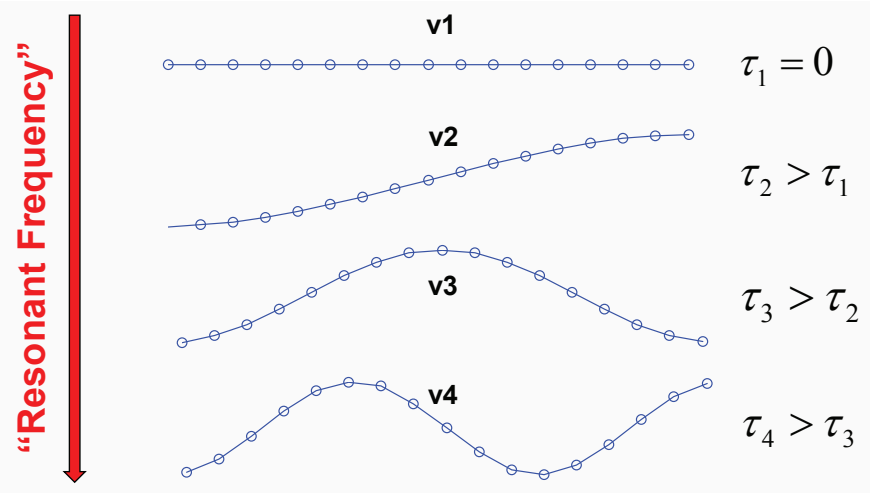

Fig. 7. The eigenvectors $\left(v_{i}\right)$ of increasing eigenvalues $\left(\tau_{i}\right)$ for a path graph.

be constructed in nearly-linear time [1], [11]. For instance, the petal-decomposition algorithm requires $O(m \log n \log \log n)$ time to generate a low-stretch spanning tree with a total stretch of $O(m \log n \log \log n)$ [1]; the $t$-step generalized power iterations in (b) can be achieved in linear time for a fixed $t$ since factorization of a tree-graph Laplacian matrix can be accomplished within linear $O(m)$ time. Consequently, the overall complexity of the proposed spectral perturbation based sparsification algorithm is almost linear.

\section{Similarity-AWARE SPECTRAL SPARSIFICATION BY EDGE FILTERING}

Although (35) and (42) provide a spectral ranking for each off-tree edge, it is not clear how many off-tree edges should be recovered to the spanning tree for achieving a desired spectral similarity level. To this end, we introduce a simple yet effective spectral off-tree edge filtering scheme motivated by recent graph signal processing techniques [26].

\section{A. Spectral Sparsification: A Low-Pass Filter on Graphs}

To more efficiently analyze signals on general undirected graphs, graph signal processing techniques have been extensively studied recently [26]. There is a clear analogy between traditional signal processing based on classical Fourier analysis and graph signal processing: 1) the signals at different time points in classical Fourier analysis correspond to the signals at different nodes in an undirected graph; 2) the more slowly oscillating functions in time domain correspond to the graph Laplacian eigenvectors associated with lower eigenvalues and more slowly varying (smoother) components across the graph. For example, the first few nontrivial eigenvectors associated with the smallest non-zero eigenvalues of a path graph Laplacian have been illustrated in Fig. 7, where the increasing eigenvalues correspond to increasing oscillation frequencies in the path graph. A comprehensive review of fundamental signal processing operations, such as filtering, translation, modulation, dilation, and down-sampling to the graph setting has been provided in [26].

Spectral sparsification aims to maintain a simplest subgraph sufficient for preserving the slowly-varying or "lowfrequency" signals on graphs, which therefore can be regarded as a "low-pass" graph filter. In other words, such spectrally sparsified graphs will be able to preserve the eigenvectors associated with low eigenvalues more accurately than high eigenvalues, and thus will retain "low-frequency" graph signals sufficiently well, but not so well for highly-oscillating (signal) components due to the missing edges.

In practice, preserving the spectral (structural) properties of the original graph within the spectral sparsifier is key to design of many fast numerical and graph-related algorithms [5], [19], [27], [30]. For example, when using spectral sparsifier as a preconditioner in preconditioned conjugate gradient (PCG) iterations, the convergence rate only depends on the spectral similarity (or relative condition number) for achieving a desired accuracy level, while in spectral graph partitioning and data clustering tasks only the first few eigenvectors associated with the smallest nontrivial eigenvalues of graph Laplacian are needed [24], [28].

\section{B. Off-Tree Edge Filtering with Joule Heat}

To only recover the off-tree edges that are most critical for achieving the desired spectral similarity level, we propose the following scheme for truncating spectrally-unique off-tree edges based on each edge's Joule heat. For a spanning-tree preconditioner, since there will be at most $k$ generalized eigenvalues that are greater than $\operatorname{st}_{P}(G) / k$, the following simple yet nearly worst-case generalized eigenvalue distribution can be assumed:

$$
\lambda_{i}=\frac{2 \lambda_{\max }}{i+1}=\frac{\operatorname{st}_{P}(G)}{i+1}, i \geq 1 .
$$

To most economically select the top- $k$ spectrally-unique offtree edges that will dominantly impact the top- $k$ largest generalized eigenvalues, the following sum of quadratic forms (Joule heat levels) can be computed based on (42) by performing $t$-step generalized power iterations with $r$ multiple random vectors $\mathbf{h}_{\mathbf{t}, \mathbf{1}}, \ldots, \mathbf{h}_{\mathbf{t}, \mathbf{r}}$ :

$$
Q_{\delta \mathbf{L}_{\mathbf{P}, \max }}\left(\mathbf{h}_{\mathbf{t}, \mathbf{1}}, \ldots, \mathbf{h}_{\mathbf{t}, \mathbf{r}}\right) \approx \sum_{j=1}^{r} \sum_{i=1}^{k}\left(\alpha_{i, j}\right)^{2}\left(\frac{2 \lambda_{\max }}{i+1}\right)^{2 t+1} .
$$

The goal is to select top $k$ spectrally-unique off-tree edges for fixing the top $k$ largest generalized eigenvalues such that the resulting upper bound of the relative condition number will become $\sigma^{2}=\frac{\tilde{\lambda}_{\max }}{\tilde{\lambda}_{\min }}$, where $\tilde{\lambda}_{\max }$ and $\tilde{\lambda}_{\min }$ denote the largest and smallest eigenvalues of $\mathbf{L}_{\mathbf{P}}^{+} \mathbf{L}_{\mathbf{G}}$ after adding top-k spectrally-unique off-tree edges. Then we have:

$$
k=2 \lambda_{\max } / \tilde{\lambda}_{\max }-1
$$

When using multiple random vectors for computing (55), it is expected that $\sum_{j=1}^{r} \alpha_{k, j}^{2} \approx \sum_{j=1}^{r} \alpha_{1, j}^{2}$, which allows us to define the normalized edge Joule heat $\theta_{k}$ for the $k$-th spectrallyunique off-tree edge through the following simplifications:

$\theta_{k}=\frac{\text { heat }_{\lambda_{k}}}{\text { heat }_{\lambda_{1}}}=\left(\frac{\sum_{j=1}^{r} \alpha_{k, j}^{2}}{\sum_{j=1}^{r} \alpha_{1, j}^{2}}\right)\left(\frac{\tilde{\lambda}_{\max }}{\lambda_{\max }}\right)^{2 t+1} \approx\left(\frac{\sigma^{2} \tilde{\lambda}_{\min }}{\lambda_{\max }}\right)^{2 t+1}$. 
The key idea of the proposed similarity-aware spectral sparsification is to leverage the normalized Joule heat (57) as a threshold for filtering off-tree edges: only the off-tree edges with normalized Joule heat values greater than $\theta_{k}$ will be selected for inclusion into the spanning tree for achieving the desired spectral similarity $(\sigma)$ level. Although the above scheme is derived for filtering spectrally-unique off-tree edges, general off-tree edges also can be filtered using similar strategies. Since adding the off-tree edges with largest Joule heat to the subgraph will mainly impact the largest generalized eigenvalues but not the smallest ones, we will assume $\tilde{\lambda}_{\text {min }} \approx \lambda_{\text {min }}$, and use the following edge truncation scheme for filtering general off-tree edges: the off-tree edge $(p, q)$ will be included into the sparsifier if its normalized Joule heat value is greater than the threshold determined by:

$$
\theta_{(p, q)}=\frac{\text { heat }_{(p, q)}}{\text { heat }_{\max }} \geq \theta_{\sigma} \approx\left(\frac{\sigma^{2} \lambda_{\min }}{\lambda_{\max }}\right)^{2 t+1}
$$

where $\theta_{\sigma}$ denotes the threshold for achieving the $\sigma$-spectral similarity in the sparsifier, and heat $t_{\max }$ denotes the maximum Joule heat of all off-tree edges computed by (35) with multiple initial random vectors.

\section{Estimation of Extreme Eigenvalues}

To achieve the above spectral off-tree edge filtering scheme, we need to compute $\theta_{\sigma}$ in (58) that further requires to estimate the extreme eigenvalues $\lambda_{\max }$ and $\lambda_{\min }$ of $\mathbf{L}_{\mathbf{P}}^{+} \mathbf{L}_{\mathbf{G}}$. In this work, we propose the following efficient methods for computing these extreme generalized eigenvalues.

1) Estimating $\lambda_{\max }$ via Power Iterations : Since generalized power iterations converge at a geometric rate determined by the separation of the two largest generalized eigenvalues $\lambda_{\max }=\lambda_{1}>\lambda_{2}$, the error of the estimated eigenvalue will decrease quickly when $\left|\lambda_{2} / \lambda_{1}\right|$ is small. It has been shown that the largest eigenvalues of $\mathbf{L}_{\mathbf{P}}^{+} \mathbf{L}_{\mathbf{G}}$ are well separated from each other [31], which thus leads to very fast convergence of generalized power iterations for estimating $\lambda_{1}$. To achieve scalable performance of power iterations, we can adopt recent graph-theoretic algebraic multigrid (AMG) methods for solving the sparsified Laplacian matrix $\mathbf{L}_{\mathbf{P}}$ [21], [41].

2) Estimating $\lambda_{\min }$ via Node Coloring: Since the smallest eigenvalues of $\mathbf{L}_{\mathbf{P}}^{+} \mathbf{L}_{\mathbf{G}}$ are crowded together [31], using (shifted) inverse power iterations may not be efficient due to the extremely slow convergence rate. To the extent of our knowledge, none of existing eigenvalue decomposition methods can efficiently compute $\lambda_{\min }$.

This work exploits the following Courant-Fischer theorem for generalized eigenvalue problems:

$$
\lambda_{\min }=\min _{|\mathbf{x}| \neq 0} \frac{\mathbf{x}^{\top} \mathbf{L}_{\mathbf{G}} \mathbf{x}}{\mathbf{x}^{\top} \mathbf{L}_{\mathbf{P}} \mathbf{x}}
$$

where $\mathbf{x}$ is also required to be orthogonal to the all-one vector. 59] indicates that if we can find a vector $\mathbf{x}$ that minimizes the ratio between the quadratic forms of the original and sparsified Laplacians, $\lambda_{\min }$ can be subsequently computed. By restricting the values in $\mathrm{x}$ to be only 1 or 0 , which can be considered as assigning one of the two colors to each node in graphs $G$ and $P$, the following simplifications can be made:

$\lambda_{\text {min }} \leq \min _{\substack{|\mathbf{x}| \neq 0 \\ x(i) \in\{0,1\}}} \frac{\mathbf{x}^{\top} \mathbf{L}_{\mathbf{G}} \mathbf{x}}{\mathbf{x}^{\top} \mathbf{L}_{\mathbf{P}} \mathbf{X}}=\min _{\substack{|\mathbf{x}| \neq 0 \\ x(i) \in\{0,1\}}} \frac{\sum_{x(p) \neq x(q),(p, q) \in E^{\prime} \neq x(q),(p, q) \in E_{s}} w_{p q}}{\sum_{p q}}$,

which will always allow estimating an upper bound for $\lambda_{\min }$. To this end, we first initialize all nodes with 0 value and subsequently try to find a node $p$ such that the ratio between quadratic forms can be minimized:

$$
\lambda_{\min } \leq \min _{p \in V} \frac{L_{G}(p, p)}{L_{P}(p, p)} .
$$

The above procedure for estimating $\lambda_{\text {min }}$ only requires finding the node with the smallest node degree ratio and thus can be easily implemented and efficiently performed for even very large graph problems. Our results for real-world graphs show that the proposed method is highly efficient and can very well estimate the smallest generalized eigenvalues when compared with existing generalized eigenvalue methods [25].

\section{Iterative Sparsification for Ill-Conditioned Problems}

To achieve more effective edge filtering for similarity-aware spectral graph sparsification, we propose to iteratively recover off-tree edges to the sparsifier through an incremental graph densification procedure. Each densification iteration adds a small portion of "filtered" off-tree edges to the latest spectral sparsifier, while the spectral similarity is estimated to determine if more off-tree edges are needed. The $i$-th graph densification iteration includes the following steps:

1) Update the subgraph Laplacian matrix $\mathbf{L}_{\mathbf{P}}$ as well as its solver by leveraging recent graph-theoretic algebraic multigrid methods [21], [41];

2) Estimate the spectral similarity by computing $\lambda_{\max }$ and $\lambda_{\min }$ using the methods described in Section IV-C,

3) If the spectral similarity is not satisfactory, continue with the following steps; otherwise, terminate the subgraph densification procedure.

4) Perform $t$-step generalized power iterations with $O(\log |V|)$ random vectors to compute the sum of Laplacian quadratic forms 55;

5) Rank and filter each off-tree edge according to its normalized Joule heat value using the threshold $\theta_{\sigma}$ in (58);

6) Check the similarity of each selected off-tree edge and only add dissimilar edges to the latest sparsifier.

\section{EXPERIMENTAL RESULTS}

The proposed spectral perturbation based spectral graph sparsification method (GRASS) has been implemented in $C++$ and available for download 2 . The proposed spectral graph sparsification algorithm allows developing nearly-linear time algorithms for tackling SDD or SDD-like sparse matrix problems, spectral graph partitioning problems, as well as graph-based regression problems [14], [19], [30], [37], [43].

\footnotetext{
${ }^{2}$ https://sites.google.com/mtu.edu/zhuofeng-graphspar
} 
TABLE I

RESULTS OF SPARSE SDD MATRIX SOLVER (SPECTRAL SPARSIFICATION WITH $5 \%$ TO $10 \%|V|$ EXTRA OFF-TREE EDGES) FOR TEST CASES IN [22].

\begin{tabular}{|c|c|c|c|c|c|c|c|}
\hline CKTs & $|V|$ & NNZ & $T_{D}\left(M_{D}\right)$ & $T_{I}\left(M_{I}\right)$ & $N_{I}$ & $\frac{\lambda_{1}}{\lambda_{1}, \text { fin }}$ & $\frac{N_{I}^{S T}}{N_{I}}$ \\
\hline ibmpg3 & $0.9 \mathrm{E} 6$ & $3.7 \mathrm{E} 6$ & $15.0 \mathrm{~s}(0.8 \mathrm{G})$ & $1.2 \mathrm{~s}(0.2 \mathrm{G})$ & 13 & $37 \mathrm{X}$ & $6 \mathrm{X}$ \\
\hline ibmpg4 & $1.0 \mathrm{E} 6$ & $4.1 \mathrm{E} 6$ & $18.3 \mathrm{~s}(1.0 \mathrm{G})$ & $1.3 \mathrm{~s}(0.2 \mathrm{G})$ & 12 & $18 \mathrm{X}$ & $4 \mathrm{X}$ \\
\hline ibmpg5 & $1.1 \mathrm{E} 6$ & $4.3 \mathrm{E} 6$ & $12.7 \mathrm{~s}(0.6 \mathrm{G})$ & $1.3 \mathrm{~s}(0.2 \mathrm{G})$ & 12 & $2,826 \mathrm{X}$ & $50 \mathrm{X}$ \\
\hline ibmpg6 & $1.7 \mathrm{E} 6$ & $6.6 \mathrm{E} 6$ & $18.3 \mathrm{~s}(0.9 \mathrm{G})$ & $2.5 \mathrm{~s}(0.3 \mathrm{G})$ & 13 & $173 \mathrm{X}$ & $13 \mathrm{X}$ \\
\hline ibmpg7 & $1.5 \mathrm{E} 6$ & $6.2 \mathrm{E} 6$ & $27.2 \mathrm{~s}(1.3 \mathrm{G})$ & $2.3 \mathrm{~s}(0.3 \mathrm{G})$ & 13 & $177 \mathrm{X}$ & $13 \mathrm{X}$ \\
\hline ibmpg8 & $1.5 \mathrm{E} 6$ & $6.2 \mathrm{E} 6$ & $18.7 \mathrm{~s}(1.3 \mathrm{G})$ & $2.3 \mathrm{~s}(0.3 \mathrm{G})$ & 13 & $120 \mathrm{X}$ & $11 \mathrm{X}$ \\
\hline
\end{tabular}

TABLE II

RESULTS OF SPARSE SDD MATRIX SOLVER (SPECTRAL SPARSIFICATION WITH $1 \%$ TO $2 \%|V|$ EXTRA OFF-TREE EDGES) FOR TEST CASES IN [35].

\begin{tabular}{|c|c|c|c|c|c|c|c|}
\hline CKTs & $|V|$ & NNZ & $T_{D}\left(M_{D}\right)$ & $T_{I}\left(M_{I}\right)$ & $N_{I}$ & $\frac{\lambda_{1}}{\lambda_{1, f i n}}$ & $\frac{N_{I}^{S T}}{N_{I}}$ \\
\hline thupg1 & $5.0 \mathrm{E} 6$ & $2.1 \mathrm{E} 7$ & $75 \mathrm{~s}(4.0 \mathrm{G})$ & $10 \mathrm{~s}(0.8 \mathrm{G})$ & 27 & $34,047 \mathrm{X}$ & $185 \mathrm{X}$ \\
\hline thupg2 & $8.9 \mathrm{E} 6$ & $3.9 \mathrm{E} 7$ & $158 \mathrm{~s}(7.6 \mathrm{G})$ & $21 \mathrm{~s}(1.5 \mathrm{G})$ & 32 & $39,426 \mathrm{X}$ & $199 \mathrm{X}$ \\
\hline thupg3 & $1.2 \mathrm{E} 7$ & $5.1 \mathrm{E} 7$ & $250 \mathrm{~s}(10.0 \mathrm{G})$ & $25 \mathrm{~s}(1.9 \mathrm{G})$ & 32 & $101,052 \mathrm{X}$ & $318 \mathrm{X}$ \\
\hline thupg4 & $1.5 \mathrm{E} 7$ & $6.6 \mathrm{E} 7$ & N/A & $36 \mathrm{~s}(2.5 \mathrm{G})$ & 32 & $97,550 \mathrm{X}$ & $312 \mathrm{X}$ \\
\hline thupg5 & $1.9 \mathrm{E} 7$ & $8.5 \mathrm{E} 7$ & N/A & $47 \mathrm{~s}(3.1 \mathrm{G})$ & 33 & $136,678 \mathrm{X}$ & $370 \mathrm{X}$ \\
\hline thupg6 & $2.4 \mathrm{E} 7$ & $1.1 \mathrm{E} 8$ & N/A & $62 \mathrm{~s}(3.8 \mathrm{G})$ & 34 & $108,898 \mathrm{X}$ & $330 \mathrm{X}$ \\
\hline thupg7 & $2.8 \mathrm{E} 7$ & $1.2 \mathrm{E} 8$ & N/A & $70 \mathrm{~s}(4.6 \mathrm{G})$ & 34 & $87,463 \mathrm{X}$ & $296 \mathrm{X}$ \\
\hline thupg8 & $4.0 \mathrm{E} 7$ & $1.8 \mathrm{E} 8$ & N/A & $110 \mathrm{~s}(6.5 \mathrm{G})$ & 34 & $368,898 \mathrm{X}$ & $607 \mathrm{X}$ \\
\hline
\end{tabular}

In this paper, a sparse SDD matrix algorithm has been implemented and compared to the state-of-the-art sparse matrix solver, Cholmod [7]. Test cases demonstrated in this paper cover a great variety of sparse SDD matrix problems obtained from realistic VLSI power grid problems [22], [35], and the sparse matrix collection from the University of Florida that includes integrated circuit simulation problems, threedimensional thermal analysis problems, finite-element analysis, etc [8]. Additionally, a spectral graph partitioning engine is also implemented, which has been dramatically accelerated by taking advantage of the proposed spectral sparsification approach. All experiments are performed using a single CPU core of a computing platform running 64-bit RHEW 6.0 with a $2.67 \mathrm{GHz}$ 12-core CPU.

\section{A. A Scalable Iterative Solver For Power Grid Analysis}

The spectral sparsifier obtained by the proposed algorithm (without weight re-scaling for the spanning tree and off-tree edges) is leveraged as a preconditioner in a PCG solver. The preconditioner is factorized by the same Cholmod solver [7]. The right-hand-side (RHS) input vector $b$ is generated randomly and the solver is set to converge to an accuracy level $\|A x-b\|<10^{-3}\|b\|$ for all test cases. " $|V|$ " denotes the number of nodes, "NNZ" denotes the number of nonzero elements in the original matrix, " $T_{D}$ " (" $T_{I}$ ") denotes the total solution time including both the matrix factorization and resolving steps of the direct (iterative) solver, " $M_{D}$ " (" $M_{I}$ ") denotes the memory cost for sparse matrix factorizations, " $N_{I}$ " denotes the number of iterations for the PCG solver to converge to the required accuracy level, $\frac{\lambda_{1}}{\lambda_{1, f i n}}$ calculates the reduction rate of the largest eigenvalue using the proposed spectral sparsification approach when compared to the initial spanning tree preconditioner, and $\frac{N_{I}^{S T}}{N_{I}}$ is the ratio of required iteration numbers using the initial spanning-tree preconditioners and the new subgraph preconditioners.
TABLE III

RESULTS OF SPARSE SDD MATRIX SOLVER (SPECTRAL SPARSIFICATION WITH $5 \%$ TO $10 \%|V|$ EXTRA OFF-TREE EDGES) FOR TEST CASES IN $|8|$.

\begin{tabular}{|c|c|c|c|c|c|c|c|}
\hline Test Cases & $|V|$ & NNZ & $T_{D}\left(M_{D}\right)$ & $T_{I}\left(M_{I}\right)$ & $N_{I}$ & $\frac{\lambda_{1}}{\lambda_{1, f i n}}$ & $\frac{N_{I}^{S T}}{N_{I}}$ \\
\hline G3_circuit & $1.6 \mathrm{E} 6$ & $7.7 \mathrm{E} 6$ & $45.1 \mathrm{~s}(2.2 \mathrm{G})$ & $5.2 \mathrm{~s}(0.3 \mathrm{G})$ & 37 & $45,897 \mathrm{X}$ & $214 \mathrm{X}$ \\
\hline thermal2 & $1.2 \mathrm{E} 6$ & $8.6 \mathrm{E} 6$ & $16.0 \mathrm{~s}(0.9 \mathrm{G})$ & $4.4 \mathrm{~s}(0.2 \mathrm{G})$ & 34 & $1,582 \mathrm{X}$ & $40 \mathrm{X}$ \\
\hline ecology2 & $1.0 \mathrm{E} 6$ & $5.0 \mathrm{E} 6$ & $12.5 \mathrm{~s}(0.7 \mathrm{G})$ & $3.6 \mathrm{~s}(0.2 \mathrm{G})$ & 47 & $1,728 \mathrm{X}$ & $42 \mathrm{X}$ \\
\hline tmt_sym & $0.7 \mathrm{E} 6$ & $5.1 \mathrm{E} 6$ & $11.8 \mathrm{~s}(0.6 \mathrm{G})$ & $2.2 \mathrm{~s}(0.1 \mathrm{G})$ & 30 & $796 \mathrm{X}$ & $28 \mathrm{X}$ \\
\hline paraboli_fem & $0.5 \mathrm{E} 6$ & $3.7 \mathrm{E} 6$ & $6.3 \mathrm{~s}(0.5 \mathrm{G})$ & $1.2 \mathrm{~s}(0.1 \mathrm{G})$ & 25 & $120 \mathrm{X}$ & $11 \mathrm{X}$ \\
\hline
\end{tabular}

TABLE IV

RESULTS OF EXTREME EIGENVALUE ESTIMATIONS.

\begin{tabular}{|c|c|c|c|c|c|c|}
\hline Test Cases & $\lambda_{\min }$ & $\tilde{\lambda}_{\min }$ & $\epsilon_{\lambda_{\min }}$ & $\lambda_{\max }$ & $\tilde{\lambda}_{\max }$ & $\epsilon_{\lambda_{\max }}$ \\
\hline fe_rotor & 1.34 & 1.40 & $4.4 \%$ & 120.9 & 116.7 & $3.5 \%$ \\
\hline pdb1HYS & 1.71 & 1.89 & $10.5 \%$ & 120.6 & 113.2 & $6.1 \%$ \\
\hline bcsstk36 & 1.18 & 1.27 & $7.6 \%$ & 96.0 & 92.4 & $3.8 \%$ \\
\hline brack2 & 1.15 & 1.20 & $4.3 \%$ & 92.6 & 90.3 & $2.5 \%$ \\
\hline raefsky3 & 1.13 & 1.25 & $10.5 \%$ & 84.4 & 82.7 & $2.0 \%$ \\
\hline
\end{tabular}

Accurate analysis of on-chip power grids is indispensable for designing modern VLSI chips since it can help reveal critical design issues related to power supply noise, electromigration, etc. However, modern power grid designs can integrate billions of components, which results in superlinear runtime/memory cost when using direct solution methods. The proposed spectral sparsification technique allows developing nearly-linear time iterative solvers for power grid analysis problems. Additionally, even more general transistorlevel SPICE-accurate circuit simulations can potentially benefit from the proposed spectral graph sparsification algorithm [14], [36], [37]. Table II and Table II] demonstrate the DC analysis results of IBM and THU power grid design benchmarks [22], [35], showing nearly-linear runtime/memory cost. Similar runtime scalability is observed from Table III for solving sparse matrices from [8]. In all test cases, the proposed spectral graph sparsification algorithm can find tree-like ultrasparsifiers with high spectral similarity. For example, we achieve $\kappa\left(L_{G}, L_{P}\right) \approx 16(\sigma \approx 4)$, for all IBM power grid test cases, which allows solving the sparse matrices within just a small number of PCG iterations (e.g. $N_{I}<14$ ).

\section{B. Estimation of Extreme Eigenvalues}

In Table IV, the extreme generalized eigenvalues $\left(\tilde{\lambda}_{\min }\right.$ and $\tilde{\lambda}_{\max }$ ) estimated by the proposed methods (Section IV-C) are compared with the ones $\left(\lambda_{\min }\right.$ and $\left.\lambda_{\max }\right)$ computed by the "eigs" function in Matlab for sparse matrices in [8], while the relative errors $\left(\epsilon_{\lambda_{\min }}\right.$ and $\left.\epsilon_{\lambda_{\max }}\right)$ are also shown. $\tilde{\lambda}_{\max }$ is estimated using less than ten generalized power iterations.

\section{Spectral Ranking of Off-tree Edges}

We illustrate the results of spectral edge ranking and filtering according to Joule heat levels computed by one-step generalized power iteration using (35) in Fig. 8 for two sparse matrices in [8]. The thresholds of normalized edge Joule heat values required for spectral edge filtering are labeled using red dash lines. It is observed in Fig. 8 there is a sharp change of the top normalized edge Joule heat values, which indicates that there are not many large eigenvalues of $\mathbf{L}_{\mathbf{P}}^{+} \mathbf{L}_{\mathbf{G}}$ in both 

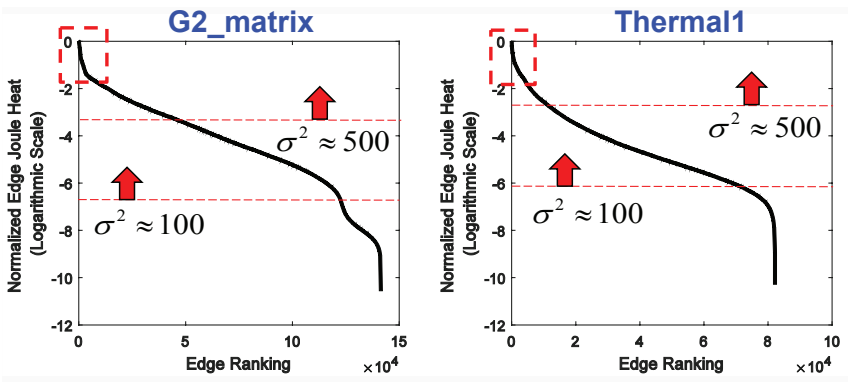

Fig. 8. Spectral edge ranking and filtering by normalized Joule heat of offtree edges for G2_circuit (left) and Thermal1 (right) test cases [8] with top off-tree edges highlighted in red rectangles.

cases and agrees well with the prior theoretical analysis [31].

We also demonstrate the results of Joule heat levels (spectral criticality) of off-tree edges computed by (35) for a random vector using one-step generalized power iteration $(t=1)$ for a "hair-comb" spanning tree shown in Fig. 9. It is not difficult to show that such a spanning tree can not well match the top part of the original 2D grid, so the Joule heat levels of offtree edges at the top should be much greater than the ones at the bottom part. In fact, it has been shown that the "haircomb" spanning tree for a $\sqrt{n} \times \sqrt{n} 2 \mathrm{D}$ mesh will have a total stretch of $\Theta(n \sqrt{n})$ that is mainly contributed by the off-tree edges with largest stretch values near the top part of the mesh grid [23]. Using the proposed similarity-aware spectral graph sparsification framework, we are able to efficiently identify and recover the most spectrally-critical off-tree edges, thereby dramatically reducing the largest generalized eigenvalues. For example, the "hair-comb" spanning tree of a $200 \times 200$ mesh grid can be dramatically improved in terms of spectral similarity by recovering top 400 spectrally-critical off-tree edges: $\sigma^{2}$ is reduced from about 64,000 to about $100(640 \times$ reduction), which can also be indicated by the Joule heat distributions before and after adding these off-tree edges as illustrated in Fig. 9

\section{Preservation of Long-Range Effects in the Sparsifier}

The similarity-aware spectral sparsifier extracted using the proposed framework will effectively preserve low-frequency graph signals or long-range effects due to the good preservation of graph spectral (structural or global) properties. As an example shown in Fig. 10, the responses of the original onchip power grid and its spectrally sparsified grid $\left(\sigma^{2}=50\right)$ are obtained by solving $\mathbf{A x}=\mathbf{b}$ and $\mathbf{A} \tilde{\mathbf{x}}=\mathbf{b}$ respectively, where $\mathbf{A}(\tilde{\mathbf{A}})$ denotes the original (sparsified) conductance matrix and b denotes a unit excitation right-hand-side (RHS) vector with only a single element being 1 and others being 0 . Note that we applied a global edge scaling procedure [40] to the sparsified power grid network in order to match the original node-wise effective resistances in $\tilde{\mathbf{A}}$. If we consider the RHS vector $b$ as the original input graph signal, and the voltage response vector $x$ as the output after graph signal processing, the power grid system can be naturally regarded as a low-pass filter for graph signals. Consequently, the solutions obtained by solving the original and sparsified power grid problems using a unit excitation source can be understood as the impulse responses of
TABLE V

RESULTS OF ITERATIVE SDD MATRIX SOLVER.

\begin{tabular}{|c|c|c|c|c|c|c|c|c|}
\hline Graphs & $|V|$ & $|E|$ & $\frac{\left|E_{50}\right|}{|V|}$ & $N_{50}$ & $T_{50}$ & $\frac{\left|E_{200}\right|}{|V|}$ & $N_{200}$ & $T_{200}$ \\
\hline G3_circuit & $1.6 \mathrm{E} 6$ & $3.0 \mathrm{E} 6$ & 1.11 & 21 & $20 \mathrm{~s}$ & 1.05 & 37 & $8 \mathrm{~s}$ \\
\hline thermal2 & $1.2 \mathrm{E} 6$ & $3.7 \mathrm{E} 6$ & 1.14 & 20 & $23 \mathrm{~s}$ & 1.06 & 36 & $9 \mathrm{~s}$ \\
\hline ecology2 & $1.0 \mathrm{E} 6$ & $2.0 \mathrm{E} 6$ & 1.14 & 20 & $16 \mathrm{~s}$ & 1.06 & 40 & $5 \mathrm{~s}$ \\
\hline tmt_sym & $0.7 \mathrm{E} 6$ & $2.2 \mathrm{E} 6$ & 1.21 & 19 & $16 \mathrm{~s}$ & 1.14 & 38 & $4 \mathrm{~s}$ \\
\hline paraboli_fem & $0.5 \mathrm{E} 6$ & $1.6 \mathrm{E} 6$ & 1.22 & 18 & $16 \mathrm{~s}$ & 1.09 & 38 & $3 \mathrm{~s}$ \\
\hline
\end{tabular}

low-pass filters commonly studied in classic Fourier analysis. Fig. 10 obviously indicates the good preservation of long range effects or low-frequency components on the spectral sparsifier obtained using the proposed method.

\section{E. An SDD Matrix Solver with Similarity-Aware Sparsification}

Fig. 11] shows the spectral drawings [18] of an airfoil graph [8] as well as its spectrally-similar $(\sigma=5)$ subgraph computed by the proposed similarity-aware spectral sparsification algorithm.

The spectral sparsifiers obtained by the proposed similarityaware algorithm are also leveraged as preconditioners in a PCG solver. The RHS input vector $b$ is generated randomly and the solver is set to converge to an accuracy level $\|\mathbf{A x}-\mathbf{b}\|<\mathbf{1 0}^{-\mathbf{3}}|| \mathbf{b} \|$ for all test cases. In Table $\mathrm{V}$. " $|V|$ " and " $|E|$ " denote the numbers of nodes and edges in the original graph, whereas " $\left|E_{\sigma^{2}}\right|$ ", " $N_{\sigma^{2}}$ " and " $T_{\sigma^{2}}$ " denote the number of edges in the sparsifier, the number of PCG iterations required for converging to the desired accuracy level, and the total time of graph sparsification for achieving the spectral similarity of $\sigma^{2}$, respectively. As observed in all test cases, there are very clear trade-offs between the graph density, computation time, and spectral similarity for all spectral sparsifiers extracted using the proposed method: sparsifiers with higher spectral similarities (smaller $\sigma^{2}$ ) allow converging to the required solution accuracy level in much fewer PCG iterations, but need to retain more edges in the subgraphs and thus require longer time to compute (sparsify).

Similar runtime scalability is observed from Table $\mathrm{V}$ for solving sparse matrices from [8]. In all test cases, the proposed spectral graph sparsification algorithm can find tree-like ultrasparsifiers with high spectral similarity. In Fig. 12, the relative residual plot (versus PCG iteration number) has been shown using three preconditioners. As observed, the PCG iterations with the preconditioner obtained by directly running the Cholesky decomposition algorithm for the spectrally-sparsified matrix computed by GRASS converge much faster than the ones with preconditioners generated by using standard incomplete Cholesky decomposition algorithm (with drop tolerances of 0.01 and 0.001 ); it is also observed that the number of nonzeros (nnz) in the preconditioner matrix created by GRASS is the lowest.

\section{F. A Scalable Spectral Graph Partitioner}

It has been shown that by applying only a few inverse power iterations, the approximate Fiedler vector $\left(u_{f}\right)$ that corresponds to the smallest nonzero eigenvalue of the (normalized) graph Laplacian matrix can be obtained for obtaining highquality graph partitioning solution [30]. Therefore, using the 

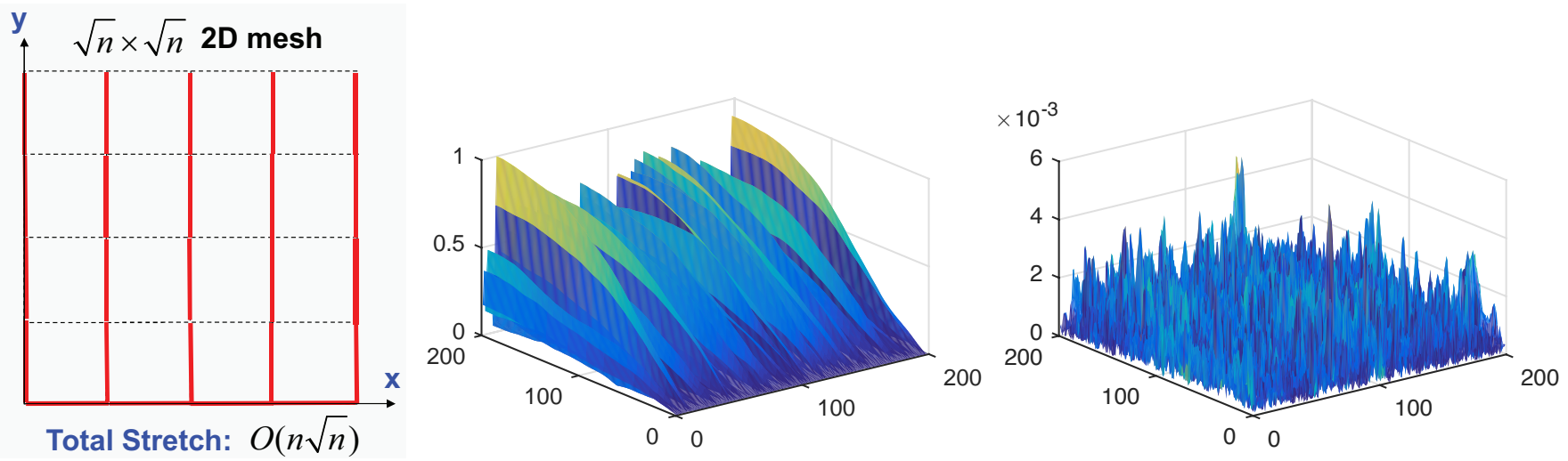

Fig. 9. Joule heat distributions before (middle) and after (rightmost) adding off-tree edges to the "hair comb" spanning tree (leftmost).

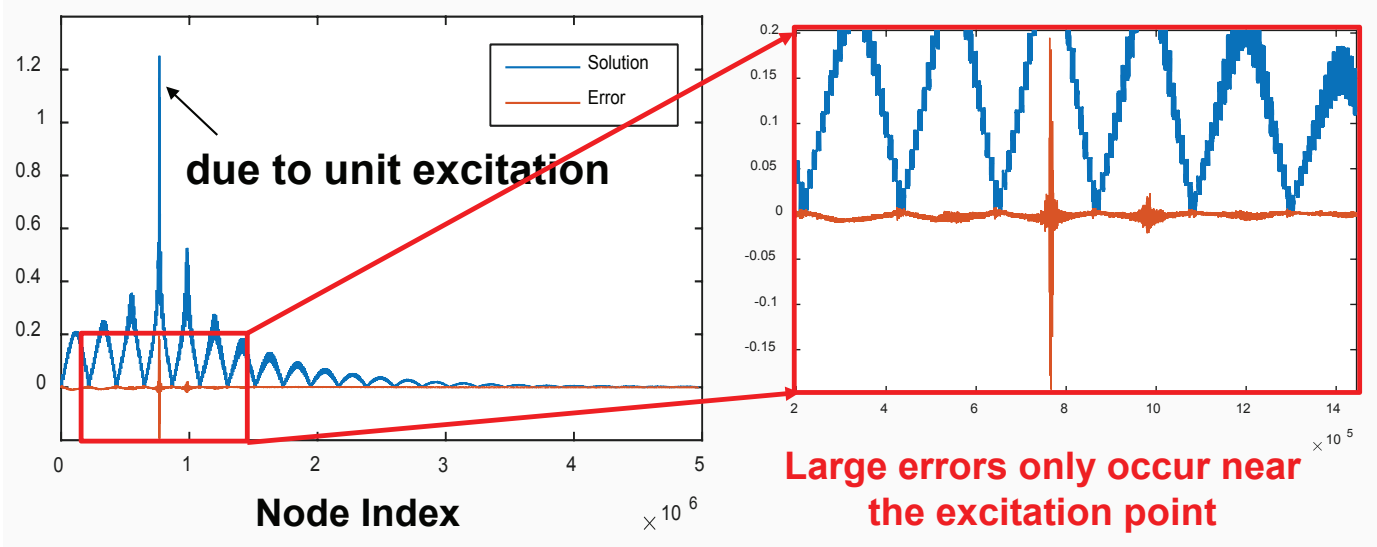

Fig. 10. Preservation of long range effects in the sparsifier.
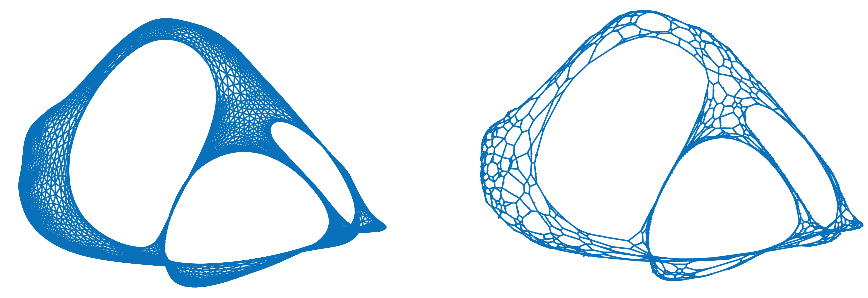

Fig. 11. Two spectrally-similar airfoil graphs $(\sigma=5)$.

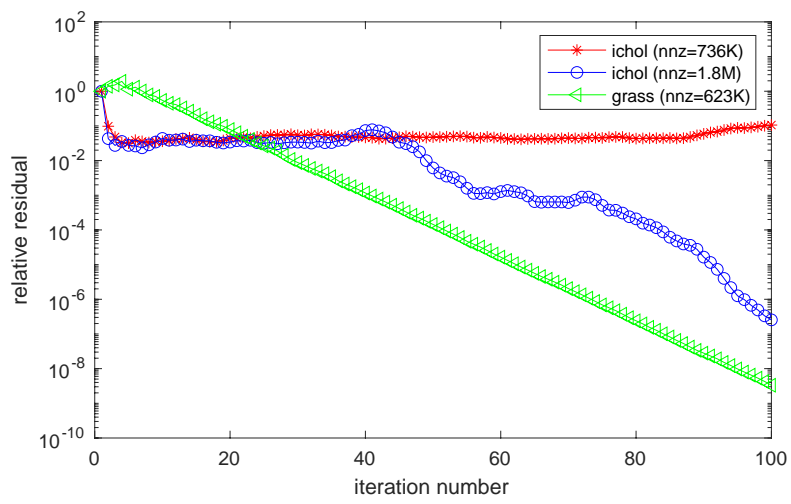

Fig. 12. PCG convergence results (G2_circuit matrix). spectral sparsifiers computed by the proposed spectral sparsification algorithm can immediately accelerate the PCG solver for inverse power iterations, leading to scalable performance for graph partitioning problems [30]. In fact, if the spectral sparsifier is already a good approximation of the original graph, its Fiedler vector can be directly used for partitioning the original graph.

We implement the accelerated spectral graph partitioning algorithm, and test it with sparse matrices in $[\overline{8}]$ and several 2D mesh graphs synthesized with random edge weights. As shown in Table VI, the graphs associated with sparse matrices have been partitioned into two pieces using sign cut method [28] according to the approximate Fiedler vectors computed by a few steps of inverse power iterations. The direct solver [7] and the preconditioned iterative solver are invoked within each inverse power iteration for updating the approximate Fiedler vectors $u_{f}$ and $\tilde{u}_{f}$, respectively. $\frac{\left|V_{+}\right|}{\left|V_{-}\right|}$denotes the ratio of nodes assigned with positive and negative signs according to the approximate Fiedler vector, and "Rel.Err." denotes the relative error of the proposed solver compared to the direct solver computed by $\frac{\left|V_{d i f}\right|}{|V|}$, where $\left|V_{\text {dif }}\right|$ denotes the number of nodes with different signs in $u_{f}$ and $\tilde{u}_{f}$. " $T_{D}$ " (" $T_{I}$ ") and " $M_{D}$ " (" $M_{I}$ ") denote the total solution time (excluding sparsification time) and memory cost of the direct (iterative) method. We extract sparsifiers with $\sigma^{2} \leq 200$ for all test cases.

It can be observed that the proposed preconditioned spectral 

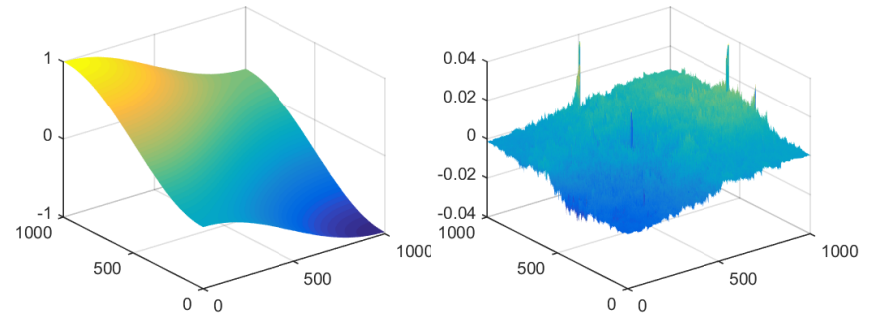

Fig. 13. The approximate Fiedler vector (left) and its magnitude error (right) for "mesh_1M".

TABLE VI

RESULTS OF SPECTRAL GRAPH PARTITIONING.

\begin{tabular}{|c|c|c|c|c|c|}
\hline Test Cases & $|V|$ & $\frac{V_{+} \mid}{\left|V_{-}\right|}$ & $T_{D}\left(M_{D}\right)$ & $T_{I}\left(M_{I}\right)$ & Rel.Err. \\
\hline G3_circuit & $1.6 \mathrm{E} 6$ & 1.35 & $52.3 \mathrm{~s}(2.3 \mathrm{G})$ & $7.6 \mathrm{~s}(0.3 \mathrm{G})$ & $2.2 \mathrm{E}-2$ \\
\hline thermal2 & $1.2 \mathrm{E} 6$ & 1.00 & $13.0 \mathrm{~s}(0.9 \mathrm{G})$ & $3.0 \mathrm{~s}(0.2 \mathrm{G})$ & $6.8 \mathrm{E}-4$ \\
\hline ecology2 & $1.0 \mathrm{E} 6$ & 1.03 & $12.1 \mathrm{~s}(0.7 \mathrm{G})$ & $3.4 \mathrm{~s}(0.2 \mathrm{G})$ & $8.9 \mathrm{E}-3$ \\
\hline tmt_sym & $0.7 \mathrm{E} 6$ & 0.99 & $10.2 \mathrm{~s}(0.6 \mathrm{G})$ & $1.9 \mathrm{~s}(0.1 \mathrm{G})$ & $2.1 \mathrm{E}-2$ \\
\hline paraboli_fem & $0.5 \mathrm{E} 6$ & 0.98 & $8.8 \mathrm{~s}(0.4 \mathrm{G})$ & $2.4 \mathrm{~s}(0.1 \mathrm{G})$ & $3.9 \mathrm{E}-2$ \\
\hline mesh_1M & $1.0 \mathrm{E} 6$ & 1.01 & $10.2 \mathrm{~s}(0.7 \mathrm{G})$ & $1.7 \mathrm{~s}(0.2 \mathrm{G})$ & $3.3 \mathrm{E}-3$ \\
\hline mesh_4M & $4.5 \mathrm{E} 6$ & 0.99 & $49.6 \mathrm{~s}(3.0 \mathrm{G})$ & $8.2 \mathrm{~s}(0.7 \mathrm{G})$ & $7.5 \mathrm{E}-3$ \\
\hline mesh_9M & $9.0 \mathrm{E} 6$ & 0.99 & $138.5 \mathrm{~s}(6.9 \mathrm{G})$ & $13.3 \mathrm{~s}(1.5 \mathrm{G})$ & $7.8 \mathrm{E}-4$ \\
\hline
\end{tabular}

graph partitioner only results in a very small portion of nodes $(0.07 \%$ to $4 \%)$ assigned with different signs when comparing with the original spectral graph partitioner, while achieving significant runtime and memory savings $(4-10 \times)$. The approximate Fiedler vector computed by our fast solver for the test case "mesh_1M" is also illustrated in Fig. 13. showing rather good agreement with the true solution.

\section{G. Sparsification of Other Complex networks}

As shown in Table VII a few finite element, protein, data and social networks have been spectrally sparsified to achieve $\sigma^{2} \approx 100$ using the proposed similarity-aware method. " $T_{t o t}$ " is the total time for extracting the sparsifier, " $\frac{\lambda_{1}}{\bar{\lambda}_{1}}$ " denotes the ratio of the largest generalized eigenvalues before and after adding off-tree edges into the spanning tree sparsifier, and $T_{\text {eig }}^{o}\left(T_{\text {eig }}^{s}\right)$ denotes the time for computing the first ten eigenvectors of the original (sparsified) graph Laplacians using the "eigs" function in Matlab. Since spectral sparsifiers can well approximate the spectral (structural) properties of the original graph, the sparsified graphs can be leveraged for accelerating many numerical and graph-related tasks. For example, spectral clustering (partitioning) using the original "RCV-80NN" (80nearest-neighbor) graph can not be performed on our server

TABLE VII

RESULTS OF COMPLEX NETWORK SPARSIFICATION.

\begin{tabular}{|c|c|c|c|c|c|c|}
\hline Test Cases & $|V|$ & $|E|$ & $T_{\text {tot }}$ & $\frac{|E|}{|E s|}$ & $\frac{\lambda_{1}}{\tilde{\lambda}_{1}}$ & $T_{\text {eig }}^{o}\left(T_{\text {eig }}^{\text {s }}\right)$ \\
\hline fe_tooth & $7.8 \mathrm{E} 4$ & $4.5 \mathrm{E} 5$ & $3.0 \mathrm{~s}$ & $5 \times$ & $8 E 3$ & $14.5 \mathrm{~s}(2.7 \mathrm{~s})$ \\
\hline appu & $1.4 \mathrm{E} 4$ & $9.2 \mathrm{E} 5$ & $5.4 \mathrm{~s}$ & $25 \times$ & $1 E 4$ & $2,400 \mathrm{~s}(15 \mathrm{~s})$ \\
\hline coAuthorsDBLP & $3.0 \mathrm{E} 5$ & $1.0 \mathrm{E} 6$ & $7.2 \mathrm{~s}$ & $3 \times$ & $1 E 3$ & $2,047 \mathrm{~s}(36 \mathrm{~s})$ \\
\hline auto & $4.5 \mathrm{E} 5$ & $3.3 \mathrm{E} 6$ & $29.0 \mathrm{~s}$ & $5 \times$ & $5 E 4$ & N/A (54s) \\
\hline RCV-80NN & $1.9 \mathrm{E} 5$ & $1.2 \mathrm{E} 7$ & $46.5 \mathrm{~s}$ & $36 \times$ & $3 E 4$ & N/A $(170 \mathrm{~s})$ \\
\hline
\end{tabular}

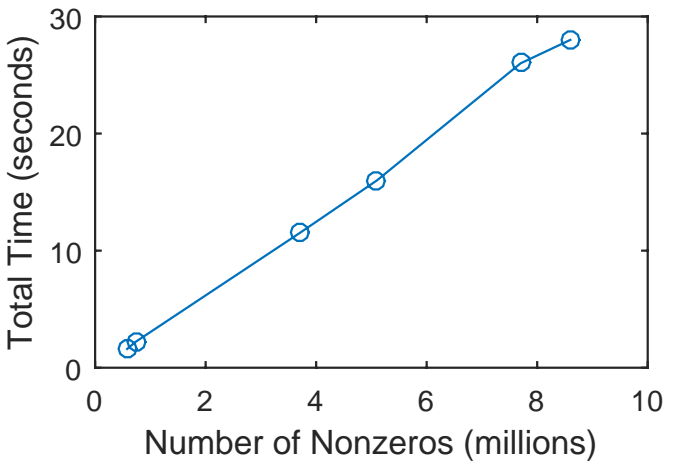

Fig. 14. Runtime scalability of the proposed similarity-aware spectral sparsification approach.

with $50 G B$ memory, while it only takes a few minutes using the sparsified one.

\section{H. Nearly-linear Runtime Scalability}

Our results show that the proposed method can extract the similarity-aware spectral sparsifier in nearly-linear time as shown in Fig. 14. It should be noted that each spectral sparsifier needs to be extracted once and can be reused or incrementally updated many times [15], [38].

\section{CONCLUSIONS}

This paper introduces a nearly-linear time yet practically efficient spectral graph sparsification algorithm that can be immediately leveraged to develop nearly-linear time sparse matrix solvers and spectral graph (data) partitioning (clustering) algorithms. A novel spectral perturbation based approach is proposed for constructing an ultra-sparse spectral graph sparsifier by adding the most spectrally-critical off-tree edges back to the initial spanning tree subgraph, so that key spectral properties of the original graph can be very well approximated. Additionally, we also propose a similarity-aware spectral graph sparsification framework that leverages efficient spectral offtree edge embedding and filtering schemes to construct spectral sparsifiers with guaranteed spectral similarity (relative condition number) level. An iterative graph densification scheme is introduced to facilitate efficient and effective filtering of off-tree edges for highly ill-conditioned problems. Extensive experimental results show the runtime of the SDD solver and the spectral graph partitioner scales nearly-linearly with the graph size for a variety of large-scale, real-world problems, such as VLSI power grid analysis, circuit simulation, finite element problems, transportation and social networks, etc. For instance, a sparse matrix with 40 million unknowns and 180 million nonzeros can be solved within two minutes using a single CPU core and about $6 G B$ memory.

\section{REFERENCES}

[1] I. Abraham and O. Neiman. Using petal-decompositions to build a low stretch spanning tree. In Proceedings of the forty-fourth annual ACM symposium on Theory of computing (STOC), pages 395-406. ACM, 2012.

[2] J. Batson, D. Spielman, and N. Srivastava. Twice-Ramanujan Sparsifiers. SIAM Journal on Computing, 41(6):1704-1721, 2012. 
[3] J. Batson, D. A. Spielman, N. Srivastava, and S.-H. Teng. Spectral sparsification of graphs: theory and algorithms. Communications of the ACM, 56(8):87-94, 2013.

[4] A. A. Benczúr and D. R. Karger. Approximating st minimum cuts in õ (n 2) time. In Proceedings of the twenty-eighth annual ACM symposium on Theory of computing (STOC), pages 47-55. ACM, 1996.

[5] P. Christiano, J. A. Kelner, A. Madry, D. A. Spielman, and S.-H Teng. Electrical flows, laplacian systems, and faster approximation of maximum flow in undirected graphs. In Proceedings of the forty-third annual ACM symposium on Theory of computing, pages 273-282, 2011.

[6] M. B. Cohen, J. Kelner, J. Peebles, R. Peng, A. B. Rao, A. Sidford, and A. Vladu. Almost-linear-time algorithms for Markov chains and new spectral primitives for directed graphs. In Proceedings of the 49th Annual ACM SIGACT Symposium on Theory of Computing, pages 410419. ACM, 2017.

[7] T. Davis. CHOLMOD: sparse supernodal Cholesky factorization and update/downdate. [Online]. Available: http://www.cise.ufl.edu/research/sparse/cholmod/, 2008.

[8] T. Davis and Y. Hu. The University of Florida sparse matrix collection. ACM Trans. on Math. Soft. (TOMS), 38(1):1, 2011.

[9] M. Defferrard, X. Bresson, and P. Vandergheynst. Convolutional neural networks on graphs with fast localized spectral filtering. In Advances in Neural Information Processing Systems, pages 3844-3852, 2016.

[10] C. Deng, Z. Zhao, Y. Wang, Z. Zhang, and Z. Feng. Graphzoom: A multi-level spectral approach for accurate and scalable graph embedding. arXiv preprint arXiv:1910.02370, 2019.

[11] M. Elkin, Y. Emek, D. Spielman, and S. Teng. Lower-stretch spanning trees. SIAM Journal on Computing, 38(2):608-628, 2008

[12] Z. Feng. Spectral graph sparsification in nearly-linear time leveraging efficient spectral perturbation analysis. In Design Automation Conference (DAC), 2016 53nd ACM/EDAC/IEEE, pages 1-6. IEEE, 2016.

[13] Z. Feng. Similarity-aware spectral sparsification by edge filtering. In Design Automation Conference (DAC), 2018 55nd ACM/EDAC/IEEE, pages 1-6. IEEE, 2018.

[14] L. Han, X. Zhao, and Z. Feng. An efficient graph sparsification approach to scalable harmonic balance (hb) analysis of strongly nonlinear $\mathrm{rf}$ circuits. In 2013 IEEE/ACM International Conference on ComputerAided Design (ICCAD), pages 494-499. IEEE, 2013.

[15] L. Han, X. Zhao, and Z. Feng. An Adaptive Graph Sparsification Approach to Scalable Harmonic Balance Analysis of Strongly Nonlinear Post-Layout RF Circuits. Computer-Aided Design of Integrated Circuits and Systems, IEEE Transactions on, 34(2):173-185, 2015.

[16] M. Imre, J. Tao, Y. Wang, Z. Zhao, Z. Feng, and C. Wang. Spectrumpreserving sparsification for visualization of big graphs. Computers \& Graphics, 87:89-102, 2020.

[17] A. Kolla, Y. Makarychev, A. Saberi, and S.-H. Teng. Subgraph sparsification and nearly optimal ultrasparsifiers. In Proceedings of the forty-second ACM symposium on Theory of computing, pages 57-66. ACM, 2010

[18] Y. Koren. On spectral graph drawing. In International Computing and Combinatorics Conference, pages 496-508. Springer, 2003.

[19] I. Koutis, G. L. Miller, and R. Peng. Approaching optimality for solving sdd linear systems. SIAM Journal on Computing, 43(1):337-354, 2014.

[20] Y. T. Lee and H. Sun. An SDP-based Algorithm for Linear-sized Spectral Sparsification. In Proceedings of the 49th Annual ACM SIGACT Symposium on Theory of Computing, STOC 2017, pages 678-687, New York, NY, USA, 2017. ACM.

[21] O. Livne and A. Brandt. Lean algebraic multigrid (LAMG): Fast graph Laplacian linear solver. SIAM Journal on Scientific Computing, 34(4):B499-B522, 2012.

[22] S. R. Nassif. IBM power grid benchmarks. [Online]. Available: http://dropzone.tamu.edu/ pli/PGBench/, 2008.

[23] R. Peng. Algorithm Design Using Spectral Graph Theory. PhD thesis, Carnegie Mellon University, 2013.

[24] R. Peng, H. Sun, and L. Zanetti. Partitioning well-clustered graphs: Spectral clustering works. In Proceedings of The 28th Conference on Learning Theory (COLT), pages 1423-1455, 2015.

[25] Y. Saad. Numerical Methods for Large Eigenvalue Problems: Revised Edition, volume 66. Siam, 2011.

[26] D. I. Shuman, S. K. Narang, P. Frossard, A. Ortega, and P. Vandergheynst. The emerging field of signal processing on graphs: Extending high-dimensional data analysis to networks and other irregular domains. IEEE Signal Processing Magazine, 30(3):83-98, 2013.

[27] D. Spielman and N. Srivastava. Graph sparsification by effective resistances. SIAM Journal on Computing, 40(6):1913-1926, 2011.

[28] D. Spielman and S. Teng. Spectral partitioning works: Planar graphs and finite element meshes. In Foundations of Computer Science (FOCS), 1996. Proceedings., 37th Annual Symposium on, pages 96-105. IEEE, 1996.
[29] D. Spielman and S. Teng. Spectral sparsification of graphs. SIAM Journal on Computing, 40(4):981-1025, 2011.

[30] D. Spielman and S. Teng. Nearly linear time algorithms for preconditioning and solving symmetric, diagonally dominant linear systems. SIAM Journal on Matrix Analysis and Applications, 35(3):835-885, 2014.

[31] D. Spielman and J. Woo. A note on preconditioning by low-stretch spanning trees. arXiv preprint arXiv:0903.2816, 2009.

[32] S.-H. Teng. Scalable algorithms for data and network analysis. Foundations and Trends ${ }^{\circledR}$ in Theoretical Computer Science, 12(1-2):1-274, 2016.

[33] Y. Wang and Z. Feng. Towards scalable spectral clustering via spectrumpreserving sparsification. arXiv preprint arXiv:1710.04584, 2017.

[34] Y. Wang, Z. Zhao, and Z. Feng. Graspel: Graph spectral learning at scale. arXiv preprint arXiv:1911.10373, 2019.

[35] J. Yang and Z. Li. THU power grid benchmarks. [Online]. Available: http://tiger.cs.tsinghua.edu.cn/PGBench/.

[36] X. Zhao and Z. Feng. Gpscp: a general-purpose support-circuit preconditioning approach to large-scale spice-accurate nonlinear circuit simulations. In 2012 IEEE/ACM International Conference on ComputerAided Design (ICCAD), pages 429-435. IEEE, 2012.

[37] X. Zhao and Z. Feng. Towards efficient spice-accurate nonlinear circuit simulation with on-the-fly support-circuit preconditioners. In Proceedings of the 49th Annual Design Automation Conference, pages 1119-1124, 2012.

[38] X. Zhao, L. Han, and Z. Feng. A Performance-Guided Graph Sparsification Approach to Scalable and Robust SPICE-Accurate Integrated Circuit Simulations. Computer-Aided Design of Integrated Circuits and Systems, IEEE Transactions on, 34(10):1639-1651, 2015.

[39] Z. Zhao and Z. Feng. A spectral graph sparsification approach to scalable vectorless power grid integrity verification. In Proceedings of the 54th Annual Design Automation Conference 2017, page 68. ACM, 2017.

[40] Z. Zhao and Z. Feng. Effective-resistance preserving spectral reduction of graphs. In Proceedings of the 56th Annual Design Automation Conference (DAC) 2019, page 109. ACM, 2019.

[41] Z. Zhao, Y. Wang, and Z. Feng. SAMG: Sparsified Graph Theoretic Algebraic Multigrid for Solving Large Symmetric Diagonally Dominant (SDD) Matrices. In Proceedings of the 36th International Conference on Computer-Aided Design (ICCAD). ACM, 2017.

[42] Z. Zhao, Y. Wang, and Z. Feng. Nearly-linear time spectral graph reduction for scalable graph partitioning and data visualization. arXiv preprint arXiv:1812.08942, 2018.

[43] X. Zhu, Z. Ghahramani, and J. D. Lafferty. Semi-supervised learning using gaussian fields and harmonic functions. In Proceedings of the 20th International conference on Machine learning (ICML-03), pages 912-919, 2003.

\begin{tabular}{|c|}
\hline \\
PLACE \\
PHOTO \\
HERE \\
\end{tabular}

Zhuo Feng (S'03-M'10-SM'13) received the B.Eng. degree in information engineering from Xi'an Jiaotong University, Xi' an, China, in 2003, the M.Eng. degree in electrical engineering from National University of Singapore, Singapore, in 2005, and the $\mathrm{Ph} . \mathrm{D}$. degree in electrical and computer engineering from Texas A\&M University, College Station, TX, in 2009 . He is currently an associate professor at Stevens Institute of Technology. His research interests include high-performance spectral methods, very large scale integration (VLSI) and computeraided design (CAD), scalable hardware and software systems, as well as heterogeneous parallel computing.

He received a Faculty Early Career Development (CAREER) Award from the National Science Foundation (NSF) in 2014, a Best Paper Award from ACM/IEEE Design Automation Conference (DAC) in 2013, and two Best Paper Award Nominations from IEEE/ACM International Conference on Computer-Aided Design (ICCAD) in 2006 and 2008. He was the principle investigator of the CUDA Research Center named by Nvidia Corporation. $\mathrm{He}$ has served on the technical program committees of major international conferences related to electronic design automation (EDA), including DAC, ASP-DAC, ISQED, and VLSI-DAT, and has been a technical referee for many leading IEEE/ACM journals in VLSI and parallel computing. In 2016, he became a co-founder of LeapLinear Solutions to provide highly scalable software solutions for solving sparse matrices and analyzing graphs (networks) with billions of elements, based on the latest breakthroughs in spectral graph theory. 